\title{
Short- and Long-Term Neurodevelopmental Outcomes of Very Preterm Infants with Neonatal Sepsis: A Systematic Review and Meta-Analysis
}

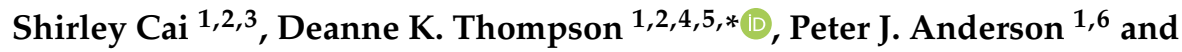 \\ Joseph Yuan-Mou Yang ${ }^{2,4,7,8}$ (D) \\ 1 Victorian Infant Brain Study, Murdoch Children's Research Institute, Royal Children's Hospital, Flemington \\ Road, Parkville, VIC 3052, Australia; shirleycai6894@gmail.com (S.C.); peter.j.anderson@monash.edu (P.J.A.) \\ 2 Developmental Imaging, Murdoch Children's Research Institute, Parkville, VIC 3052, Australia; \\ Joseph.Yang4@rch.org.au \\ 3 Melbourne Medicine School, Faculty of Medicine, Dentistry and Health Sciences, The University of \\ Melbourne, Parkville, VIC 3052, Australia \\ 4 Department of Paediatrics, The University of Melbourne, Parkville, VIC 3052, Australia \\ 5 Florey Institute of Neuroscience and Mental Health, Parkville, VIC 3052, Australia \\ 6 Turner Institute for Brain and Mental Health, School of Psychological Sciences, Monash University, Clayton, \\ VIC 3800, Australia \\ 7 Neuroscience Research, Murdoch Children's Research Institute, Parkville, VIC 3052, Australia \\ 8 Department of Neurosurgery, Royal Children's Hospital, Parkville, VIC 3052, Australia \\ * Correspondence: deanne.thompson@mcri.edu.au
}

Received: 7 November 2019; Accepted: 23 November 2019; Published: 1 December 2019

\begin{abstract}
Sepsis is commonly experienced by infants born very preterm ( $<32$ weeks gestational age and/or $<1500 \mathrm{~g}$ birthweight), but the long-term functional outcomes are unclear. The objective of this systematic review was to identify observational studies comparing neurodevelopmental outcomes in very preterm infants who had blood culture-proven neonatal sepsis with those without sepsis. Twenty-four studies were identified, of which 19 used prespecified definitions of neurodevelopmental impairment and five reported neurodevelopmental outcomes as continuous variables. Meta-analysis was conducted using 14 studies with defined neurodevelopmental impairment and demonstrated that very preterm infants with neonatal sepsis were at higher risk of impairments, such as cerebral palsy and neurosensory deficits, compared with infants without sepsis (OR 3.18; 95\% CI 2.29-4.41). Substantial heterogeneity existed across the studies $\left(I^{2}=83.1,95 \%\right.$ CI 73-89). The five studies that reported outcomes as continuous variables showed no significant difference in cognitive performance between sepsis and non-sepsis groups. Neonatal sepsis in very preterm infants is associated with increased risk of neurodevelopmental disability. Due to the paucity of longitudinal follow-up data beyond 36 months, the long-term cognitive effect of neonatal sepsis in very preterm infants could not be conclusively determined. Effects on the development of minor impairment could not be assessed, due to the small numbers of infants included in the studies.
\end{abstract}

Keywords: premature; brain; infection; infant; development; cognition

\section{Introduction}

Sepsis is a clinical condition characterised by bacteraemia and clinical signs of systemic infection [1]. It is one of the events that can occur during neonatal intensive care unit (NICU) admission and contributes significantly to the morbidity of very preterm (VP: $<32$ weeks gestational age) and/or very low birthweight (VLBW: $<1500 \mathrm{~g}$ ) infants [2-4]. Studies have reported rates of sepsis are inversely 
proportional to gestational age, with $33 \%$ of infants born less than 28 weeks acquiring sepsis compared with $60 \%$ of infants born less than 25 weeks [5].

During the neonatal period, complications such as sepsis can have dramatic effects on the growth and development of the child, especially in children born very prematurely [6,7]. The mechanism of how sepsis inflicts brain damage has been hypothesised. Research suggests the developing brain is vulnerable to the systematic inflammatory milieu characteristic of sepsis, as well as cytotoxic and ischaemic injury from hypotension and reduced cerebral blood flow [7]. Together, these factors may result in white matter abnormalities and diffuse injury to premyelinating oligodendrocytes, which have been shown to be closely associated with increased risk for impaired cognitive and motor functioning [8,9]. Such morbidities are complex and can range from major impairments, such as cerebral palsy, to more subtle deficits such as difficulties with memory and attention. Regardless, they all have the potential to affect a child's academic, social and emotional functioning. Neurodevelopmental follow-up in VP infants with sepsis is crucial for the early identification of developmental delay so that targeted interventions can be prescribed to minimise long-lasting impairments.

The short-term cognitive impact of sepsis in VP infants has been studied. A systematic review of 17 studies on VLBW infants with neonatal sepsis concluded these infants are at twice the risk of neurodevelopmental impairment compared with their non-sepsis counterparts [10]. The follow-up duration of studies included in this review ranged from 6 to 60 months; 11 of the 17 studies had a follow-up duration of less than 36 months. Many studies focused on serious disabilities, such as cerebral palsy and neurosensory impairment, using dichotomised definitions of neurodevelopmental impairment (NDI) that were study-specific. Little is known about NDI beyond 36 months and whether they impact other cognitive domains.

A comprehensive analysis of the long-term consequences of sepsis in children born VP is needed to better inform parents and health professionals of the long-term cognitive risks and to guide targeted and age-specific interventions in high-risk infants to achieve optimal function.

The objective of this systematic review was to provide an updated review of all VP cohort studies, which evaluated long-term neurodevelopmental outcomes beyond 18 months of age in infants who had culture-positive neonatal sepsis compared with VP infants without sepsis.

\section{Materials and Methods}

The review protocol consisted of a detailed research question, search strategy, initial screening criteria and full-text screening criteria. Using the PICOS (population, intervention, comparator, outcome, study design) approach, the research question was formulated as follows: "Does postnatal sepsis in VP infants adversely impact long-term neurodevelopmental outcomes compared with VP infants without sepsis exposure?".

\subsection{Literature Search Strategy}

An electronic search strategy was used to identify relevant studies in the following databases by one reviewer (S.C.): Medline (using Ovid, 1966-present), Embase (using Ovid, 1980-present) and PubMed (1966-present). The search strategy for the Medline database comprised the following Medical Subject Headings (MeSH) or keywords, using Boolean terms 'or' then combined with 'and': (1) infant, premature or infant, low birthweight; (2) and sepsis; (3) and neurodevelopmental disorders or developmental disabilities or intellectual disability or learning disorders or motor skills disorders. The search was then adapted for each database and the last search was conducted on 24 February 2018, see Table S1 for the full search strategy. Additional article screening was performed by hand search of the reference lists of selected articles.

\subsection{Study Selection Criteria}

After removing duplicated studies, the search results were screened using titles and abstracts. Full texts of the selected articles were then reviewed independently by one reviewer (S.C.). The articles 
were included if they were full-text academic journal articles that reported quantitative information on our PICOS parameters in observational clinical studies.

Studies had to meet the following selection criteria for inclusion: (1) Participants comprised of infants born $<32$ weeks' gestational age and/or $<1500 \mathrm{~g}$ birth weight with no major genetic or congenital abnormalities; (2) blood culture-proven sepsis during the NICU admission, with optional additional criteria for sepsis definition as per study; and (3) follow-up duration of at least 18 months assessing neurodevelopment. We intended to include studies with a longer follow-up duration, starting at 18 months, and had no restriction on the upper limit of follow-up duration.

Additional study inclusion criteria included: (1) Studies reporting original data; (2) if there were studies that used the same outcome data from the same institution, the one with greater information was included; (3) studies that included VP infants with sepsis as part of a larger preterm cohort and contained sufficient outcome information; (4) studies published in English or had been translated into English. Study exclusion criteria included: (1) Review articles, nonanalytical studies and expert opinion articles; (2) studies where outcomes were not reported; (3) studies where outcomes of the sepsis exposure group could not be separated from those of the non-sepsis exposure group. No restriction on publication or publication status was applied.

\subsection{Data Extraction}

The information extracted from studies included: study design (single vs multi-centre; case-control vs. case-cohort studies), year of birth, number of infants with and without sepsis, micro-organism isolated from blood cultures, age at follow-up and blinding status of outcome assessors. This review only included infants with sepsis and excluded any cases with suspected sepsis or necrotising enterocolitis. For each study, data from neurodevelopmental assessment tools and relevant NDI definitions were tabulated. For studies with defined dichotomous outcomes as per study NDI definition, the number of infants with NDI in the sepsis and the non-sepsis groups were calculated. In studies where the odds ratio (OR) for association was reported, individual numbers were calculated using the $2 \times 2$ contingency tables. For individual studies with reported neurodevelopmental outcome assessments as continuous variables, the mean values and standard deviations (SD) for the sepsis and non-sepsis group were recorded. All numbers used were unadjusted.

\subsection{Assess Risk of Bias in Individual Studies}

Each study was assessed for the risk of bias using a modified version of the Cochrane Collaboration's tool for assessing risk of bias, as seen in Table S2 [11]. Each study was examined for selection bias, performance bias, attrition bias, detection bias and reporting bias and ranked as 'low risk' or 'high risk'. It was marked 'unclear' if there was insufficient information to make an assessment.

\subsection{Data Synthesis and Analysis}

STATA version 15 (Stata, College Station, TX, USA) and the STATA meta-analysis software package 'metan' were used for all statistical analysis. The meta-analysis was conducted using a random-effect model based on the DerSimonian \& Laid method [12,13]. Results were reported only from the random-effect model, given the data from the literature was expected to have variable effect size. For studies reporting dichotomous outcome variables (i.e., NDI vs. no NDI), estimates for OR and their corresponding 95\% confidence interval (CI) and percentage weight contributing to the overall meta-analysis from each study were calculated. The impact of missing outcome data was explored using a variety of scenarios including available case analysis, best-case scenario, worst-case scenario and the informative missingness model [14]. For studies reporting continuous outcome variables, the mean and SD were compared. Due to the diversity of neurocognitive tools and domains assessed, the results of studies reporting continuous outcome variables were unable to be pooled for meta-analysis.

The inconsistency of effects across studies was measured by estimating statistical heterogeneity across included studies using the $\mathrm{I}^{2}$ calculation along with the associated $95 \% \mathrm{CI}[15,16]$. The advantage 
of this measure of inconsistency is that it does not depend on the number of studies and is accompanied by an uncertainty interval - the predictive interval —which shows the predictive distribution of a future trial, based on the extent of heterogeneity [17]. $p<0.05$ defined statistical significance in the heterogeneity analysis.

To assess the possibility of publication bias, the log transform of the effect size was plotted against the inverse of its standard error to generate a contour-enhanced funnel plot [18]. The plots were visually inspected for asymmetry of data points, which may represent publication bias. Egger's meta-regression test was performed to examine small study effect to see if the effect decreased with increasing sample size [19]. A subgroup analysis of studies with a follow-up duration of 36 months or greater was performed to investigate long-term NDI.

\section{Results}

\subsection{Study Selection}

The study selection process is summarised in Figure 1. Database searching identified 1165 articles. After removing duplicates, 727 articles were available for screening. A total of 680 were excluded as they did not meet the initial selection criteria. After full-text assessment of the remaining 47 articles, another 23 were excluded [20-42]. The remaining 24 articles were eligible for data analysis and synthesis. Of these, 14 were eligible for quantitative synthesis. The remaining ten studies were analysed via qualitative synthesis. Hand searching through the reference lists of the included articles yielded one additional study which was later excluded during full-text analysis [42].

\subsection{Study Characteristics}

Twenty-four studies met the study selection criteria. The details of the study design and study population are summarised in Table 1. Eight studies were multicentre cohort studies [43-50], 12 were single-centre cohort studies [51-62] and four were single-centre case-control studies [63-66]. All studies were retrospective in design. Ten studies used retrospectively collected data [51-53,56,59,60,63-65] and 13 studies used prospectively collected data $[44-50,55,57,58,61,62,66]$. Publications years were between 1994 and 2019. 


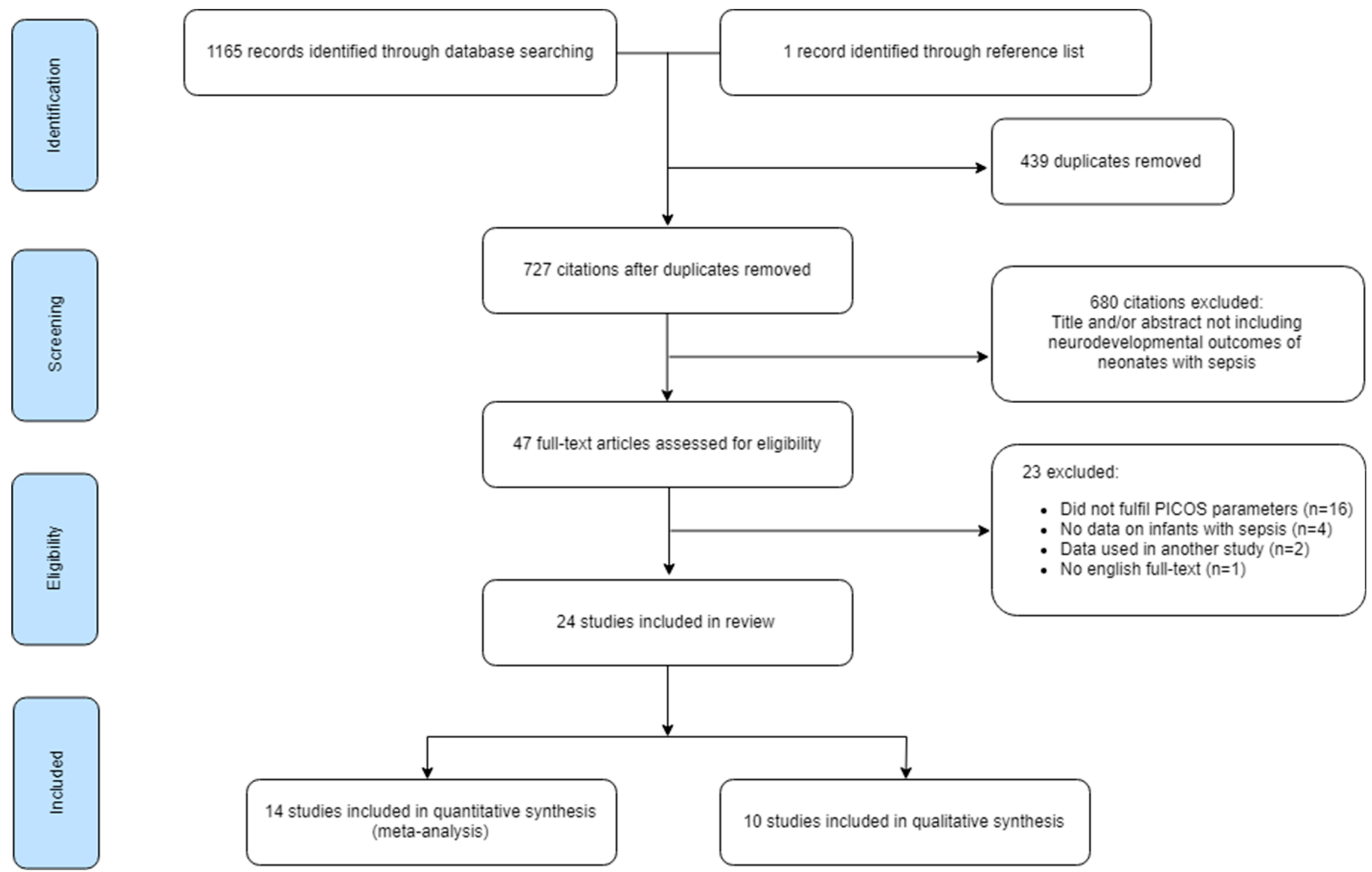

Figure 1. Flow diagram of the study selection process. 
Table 1. Summary of the study design and study population characteristics of all included studies.

\begin{tabular}{|c|c|c|c|c|c|c|c|c|c|c|c|c|}
\hline Author & Year & Study Design & Population & Birth Year & $\begin{array}{c}\text { No. of } \\
\text { Survivors at } \\
\text { Follow-Up }\end{array}$ & $\begin{array}{c}\text { Follow-Up } \\
\text { Rate }\end{array}$ & $\begin{array}{c}\text { Total No. } \\
\text { Followed Up }\end{array}$ & $\begin{array}{c}\text { No. of } \\
\text { Confirmed } \\
\text { Sepsis }\end{array}$ & $\begin{array}{c}\text { No. of } \\
\text { Non-sepsis } \\
\text { Comparators }\end{array}$ & $\begin{array}{l}\text { Organism } \\
\text { Isolated }\end{array}$ & $\begin{array}{l}\text { Age at } \\
\text { Assessment } \\
\text { (Months) }\end{array}$ & $\begin{array}{c}\text { Blinding o } \\
\text { Outcome } \\
\text { Assessors }\end{array}$ \\
\hline Msall [51] & 1994 & SC, cohort & GA 23-28 & 1983-1986 & 153 & $97 \%$ & 149 & 18 & 131 & NS & $52-62$ & Yes \\
\hline Lee [63] & 1998 & $\mathrm{SC}$, case control & $\mathrm{BW}<1250$ & 1990-1995 & 35 & N/A & N/A & 14 & 21 & Candida & $9-50$ & NS \\
\hline Friedman [52] & 2000 & SC, cohort & ELBW & 1988-1996 & 334 & $90 \%$ & 299 & 27 & 272 & Candida & $21-24$ & NS \\
\hline Hack [53] & 2000 & SC, cohort & ELBW & 1992-1995 & 241 & $92 \%$ & 221 & 109 & 112 & NS & 20 & NS \\
\hline Hoekstra [54] & 2004 & SC, cohort & GA $23-26$ & 1986-2000 & 778 & $87 \%$ & 675 & NS & NS & NS & $36-60$ & No \\
\hline Stoll [43] & 2004 & MC, cohort & ELBW & 1993-2001 & 7892 & $80 \%$ & 6314 & 1922 & 2161 & $\begin{array}{c}\text { Shows } \\
\text { breakdown }\end{array}$ & $18-22$ & NS \\
\hline Shah [55] & 2008 & SC, cohort & $\mathrm{GA}<30$ & 2001-2003 & 204 & $94 \%$ & 192 & 64 & 119 & NS & 24 & NS \\
\hline Kono [44] & 2011 & $\mathrm{MC}$, cohort & VLBW & 2003-2004 & 2847 & $64 \%$ & 1826 & 113 & 1714 & NS & $36-42$ & NS \\
\hline Jang [56] & 2011 & SC, cohort & VLBW & 1989-2007 & 967 & N/A & N/A & NS & NS & NS & $18-24$ & NS \\
\hline Schlapbach [45] & 2011 & MC, cohort & GA $24-27$ & 2000-2007 & 482 & $77 \%$ & 372 & 136 & 236 & $\begin{array}{c}\text { Shows } \\
\text { breakdown }\end{array}$ & $18-24$ & NS \\
\hline Van der Ree [64] & 2011 & SC, case-control & $\begin{array}{l}\text { GA }<32 \text { or } \\
\text { VLBW }\end{array}$ & 2000-2001 & 50 & N/A & N/A & 32 & 18 & $\begin{array}{c}\text { Shows } \\
\text { breakdown }\end{array}$ & 72-108 & NS \\
\hline $\begin{array}{c}\text { Adams-Chapman } \\
{[46]}\end{array}$ & 2013 & MC, cohort & ELBW & 2004-2007 & 1966 & $71 \%$ & 1391 & 474 & 917 & $\begin{array}{c}\text { Candida and } \\
\text { other }\end{array}$ & $18-22$ & NS \\
\hline De Haan [65] & 2013 & SC, case-control & $\mathrm{GA}<30$ & 1997-2007 & 168 & $90 \%$ & 152 & 50 & 102 & $\begin{array}{l}\text { GN and } \\
\text { Candida }\end{array}$ & 24 & NS \\
\hline Dilli [66] & 2013 & SC, case control & VLBW & 2008-2009 & 33 & N/A & N/A & 13 & 20 & NS & $18-24$ & NS \\
\hline Mitha [47] & 2013 & MC, cohort & GA 22-32 & 1997 & 2277 & $78 \%$ & 1769 & 688 & 1081 & NS & 60 & NS \\
\hline Alshaikh [57] & 2014 & SC, cohort & GA $<29$ & 1995-2008 & 383 & $87 \%$ & 332 & 105 & 227 & CONS & $30-42$ & Yes \\
\hline Hentges [58] & 2014 & SC, cohort & $\begin{array}{l}\text { GA }<32 \text { or } \\
\text { VLBW }\end{array}$ & 2003-2010 & 291 & $78 \%$ & 226 & 62 & 164 & $\begin{array}{c}\text { Shows } \\
\text { breakdown }\end{array}$ & $18-24$ & Yes \\
\hline Yang [59] & 2015 & SC, cohort & VLBW & 1996-1999 & 111 & $55 \%$ & 61 & 26 & 35 & NS & $144-180$ & NS \\
\hline Maruyama [60] & 2016 & SC, cohort & VLBW & 2005-2012 & 200 & $78 \%$ & 155 & N/A & N/A & NS & $36-42$ & NS \\
\hline Synnes [48] & 2016 & MC, cohort & GA $<29$ & 2009-2011 & 2340 & $80 \%$ & 1870 & NS & NS & NS & $18-21$ & NS \\
\hline Young [61] & 2016 & SC, cohort & GA $<32$ & 2008-2010 & 100 & N/A & N/A & 17 & 33 & NS & 24,48 & NS \\
\hline Bright [49] & 2017 & MC, cohort & $\mathrm{GA}<28$ & 2002-2004 & 966 & $92 \%$ & 889 & 223 & 532 & NS & 120 & Yes \\
\hline Bolisetty [50] & 2018 & MC, cohort & GA 23-28 & $2007-2012$ & 1897 & $80 \%$ & 1514 & 526 & 988 & NS & $24-36$ & NS \\
\hline Zonnenberg [62] & 2019 & SC, cohort & $\begin{array}{c}\mathrm{GA}<32 \text { or } \\
\mathrm{VLBW}\end{array}$ & 2008-2014 & 104 & $87 \%$ & 90 & 68 & 22 & $\begin{array}{c}\text { Shows } \\
\text { breakdown }\end{array}$ & 24 & Yes \\
\hline
\end{tabular}

Abbreviations: SC—-single-centre, MC—-multicentre, GA—gestational age (weeks), BW—birth weight (grams), ELBW—extremely low birth weight (<1000 g), VLBW—very low birth

weight $(<1500 \mathrm{~g})$, GN—Gram-negative organisms, CONS—coagulase negative Staphylococci, N/A—not applicable, NS—not specified. 
Overall, the total number of survivors at follow-up ranged from 33 to 7892, with the number of confirmed sepsis ranging from 13 to 1922 and the number of non-sepsis comparators ranging from 18 to 2161 . Birth years were between 1983 and 2014. Overall, the median attrition rate of all studies was 20\% (range $3 \%-45 \%)$. Fourteen studies specifically analysed the impact of sepsis $[45-47,49,52,55,57,58,62-66]$, whereas the other ten studied perinatal variables more generally with sepsis as a factor $[44,48,50,51,53,56,59-61]$. Fourteen studies did not report the prevalence of each micro-organism [44,47-51,53-56,59-61,66]. Seven analysed the prevalence of each microorganism $[43,45,46,58,62,64,65]$, of which four included fungal infections $[43,46,58,65]$. Two studies restricted their studies to Candidaemia only $[52,63]$ and one restricted to coagulase-negative Staphylocci sepsis only [57]. Follow-up duration ranged from nine to 180 months. Four studies had a follow-up duration of more than or equal to 36 months $[47,51,59,60]$. Five studies had blinded outcome assessors [49,51,57,58,62], one reported no blinding was performed [54] and the rest did not specify [43-46,48,50,52,53,55,56,59-61,63-66]. Age of assessment ranged from 18 months to 15 years. All studies contained information on the neurodevelopmental assessment tools used and details of neurodevelopmental outcomes assessed as summarised in Table 2. Nineteen studies reported dichotomised outcomes using a definition for NDI $[43-54,56,57,59,60,63,65,66]$, whereas the other five reported outcomes as continuous variables $[55,58,61,62,64]$.

\subsection{Risk of Bias within Studies}

Figure 2 summarises the risk of bias from all studies; see Table S3 for detail assessment from each study. Overall, six studies were ranked high risk and 18 studies were ranked low risk for selection bias. All studies were ranked low risk for performance bias. Eleven studies were ranked high risk and 13 studies were ranked low risk for attrition bias. Two studies were ranked high risk and four studies were ranked low risk for detection bias, with the remaining 18 marked as unclear. Four studies were ranked high risk and 20 studies were ranked low risk for reporting bias.

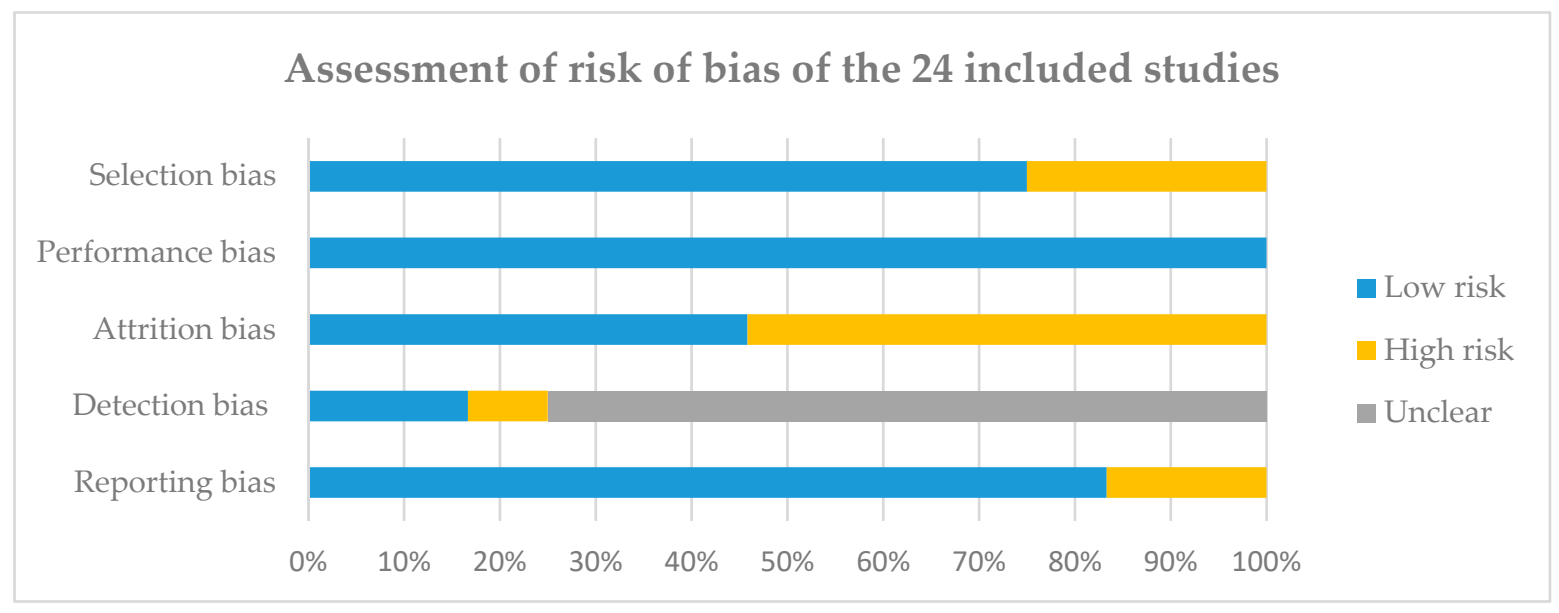

Figure 2. Assessment of risk of bias of the 24 included studies. The risk of bias was assessed using a modified version of the Cochrane Collaboration's tool for assessing risk of bias; see Table S2. 
Table 2. Neurodevelopmental outcome scales used by the included studies and their respective neurodevelopmental impairment definitions.

\begin{tabular}{|c|c|c|}
\hline Study & Scale Used & NDI Definitions \\
\hline \multicolumn{3}{|c|}{ Studies Which Reported Dichotomised Outcomes } \\
\hline \multirow[b]{2}{*}{ Msall [51] } & \multicolumn{2}{|r|}{ One or more of the following: } \\
\hline & $\begin{array}{l}\text { McCarthy Scales of Children's Abilities, Cattell Infant } \\
\text { Intelligence Test or the Clinical Linguistic Auditory Milestone } \\
\text { Scale (for children with a mental age }<30 \text { months) }\end{array}$ & $\begin{array}{l}\text { - CP defined as early onset, nonprogressive motor and postural delay (hemiplegia, } \\
\text { diplegia or quadriplegia) } \\
\text { - } \quad \text { IQ: Mild 52-67, Moderate 36-51, Severe 20-35 on McCarthy instrument } \\
\text { - } \quad \text { Blindness defined as legal blindness or corrected vision worse than 20/200 } \\
\text { - } \quad \text { Deafness defined as bilateral hearing impairment of more than } 85 \mathrm{~dB}\end{array}$ \\
\hline & \multicolumn{2}{|r|}{ One or more of the following: } \\
\hline Lee [63] & $\begin{array}{c}\text { BSID-II (<24 months), Stanford-Binet Intelligence Scale, } \\
\text { Peabody Developmental Motor Scales }\end{array}$ & $\begin{array}{ll}\text { - } & \text { CP of all types of severity } \\
\text { - } & \text { Legal blindness (corrected visual acuity of the better eye, }<20 / 200) \\
\text { - } & \text { Hearing loss (neurosensory hearing loss in the better ear, }>30 \mathrm{~dB} \text { ) } \\
\text { - } & \text { Cognitive delay (MDI, }>3 \text { SD below the mean) }\end{array}$ \\
\hline Friedman [52] & BSID-II & $\begin{array}{l}\text { Mild-moderate: mild-moderate CP, moderate cognitive delay (MDI 70-82) } \\
\text { Severe: severe CP (not sitting by } 2 \text { years of age, non-ambulatory), bilateral blindness, aided } \\
\text { sensorineural hearing loss, severe cognitive delay (MDI < 70), shunted hydrocephalus }\end{array}$ \\
\hline \multirow[b]{2}{*}{ Hack [53] } & \multirow[b]{2}{*}{ BSID-II } & One or more of the following: \\
\hline & & $\begin{array}{l}\text { - CP (spastic diplegia, hemiplegia, hemiplegia, or quadriplegia), hypertonia, hypotonia } \\
\text { and shunt-dependent hydrocephalus } \\
\text { - } \quad \text { Blindness unilateral or bilateral } \\
\text { - } \quad \text { Deafness unilateral or bilateral } \\
\text { - } \quad \text { MDI }<70\end{array}$ \\
\hline \multirow[t]{3}{*}{ Hoekstra [54] } & BSID-II ( $<36$ mo $)$ & $\begin{array}{l}\text { Mild-moderate: isolated muscle tone abnormalities, unilateral blindness, hyperactivity, scores } \\
\text { 1-2 SD below mean } \\
\text { Severe: spasticity, severe hypotonia, blindness, deafness, scores }>2 \text { SD below mean }\end{array}$ \\
\hline & $\begin{array}{c}\text { 3-6 years: Denver Developmental Screening Test, Early } \\
\text { Language Milestone Scale, Zimmerman Preschool } \\
\text { Articulation Test }\end{array}$ & $\begin{array}{l}\text { Mild-moderate: minor abnormalities, developmental assessments 6-12 months below } \\
\text { chronological age } \\
\text { Severe: severe physical and neurologic examinations, developmental assessments }>1 \text { year } \\
\text { below chronological age }\end{array}$ \\
\hline & $\begin{array}{l}\text { School children: University of Vermont Achenbach Child } \\
\text { Behaviour Checklist and the Teacher's Report form }\end{array}$ & $\begin{array}{l}\text { Mild-moderate: below grade average in }>1 \text { subject, but not far below in }>1 \text { subject, or had } \\
\text { below-normal Achenbach scores } \\
\text { Severe: spasticity, severe hypotonia, blindness, hearing loss, repeated a grade, require special } \\
\text { education, far below grade average in }>1 \text { subject or Achenbach scores far below normal }\end{array}$ \\
\hline
\end{tabular}


Table 2. Cont.

\begin{tabular}{|c|c|c|}
\hline Study & Scale Used & NDI Definitions \\
\hline Stoll [43] & BSID-II & $\begin{array}{l}\text { One or more of the following: } \\
-\quad \mathrm{CP} \\
-\quad \mathrm{MDI}<70 \text { or PDI }<70 \\
-\quad \text { Bilateral blindness } \\
-\quad \text { Bilateral hearing impairment }\end{array}$ \\
\hline Kono [44] & KPSD & $\begin{array}{l}\text { One or more of the following: } \\
\text { - } \quad \mathrm{CP} \text { (nonprogressive } \mathrm{CNS} \text { disorder characterised by abnormal muscle tone in at least one } \\
\text { extremity and abnormal control of movement and posture) } \\
\text { - } \quad \text { Unilateral or bilateral blindness } \\
\text { - } \quad \text { Hearing impairment requiring hearing aids } \\
\text { - } \quad \text { KPSD }<70 \text { or judged by physicians (i.e., ability to say any meaningful words, ability to } \\
\text { say own name or age, able to build using several small bricks, able to distinguish size of } \\
\text { circles with a diameter of } 4 \mathrm{~cm} \text { and } 6 \mathrm{~cm} \text { ) }\end{array}$ \\
\hline Jang [56] & BSID or Denver Developmental Screening Test & $\begin{array}{l}\text { One or more of the following: } \\
\text { - } \mathrm{CP} \text { (permanent, but no unchanging, disorder of movement and/or posture and of motor } \\
\text { function caused by a nonprogressive interference, lesion, or abnormality of the } \\
\text { developing immature brain) } \\
>6 \text { months delay of motor and/or mental development including cognitive impairment, } \\
\text { psychomotor impairment and neurosensory impairment }\end{array}$ \\
\hline Schlapbach [45] & BSID-II, GMFCS & $\begin{array}{l}\text { One or more of the following: } \\
\text { - } \quad \mathrm{CP} \text { (nonprogressive motor disorder characterised by abnormal tone in at least one } \\
\text { extremity and abnormal control of movement and posture) } \\
\text { - } \quad \mathrm{MDI}<70 \text { or } \mathrm{PDI}<70 \\
\text { - } \quad \text { Bilateral blindness } \\
\text { - } \quad \text { Hearing impairment requiring amplification }\end{array}$ \\
\hline
\end{tabular}


Table 2. Cont

\begin{tabular}{|c|c|c|}
\hline Study & Scale Used & NDI Definitions \\
\hline Adams-Chapman [46] & BSID-II, BSID-III, GMFCS & $\begin{array}{ll}\text { BSID-II (Epoch 1) } \\
-\quad & \text { Moderate-severe CP with GMFCS } \geq 2 \\
- & \text { MDI }<70 \text { or PDI }<70 \\
- & \text { Bilateral blindness with no functional vision } \\
- & \text { Bilateral amplification for permanent hearing loss } \\
\text { BSID-III (Epoch 2) } \\
-\quad \\
\text { Moderate to severe CP with GMFCS } \geq 2 \\
-\quad \text { BSID-III cognitive }<70 \\
-\quad \text { Visual acuity }<20 / 200 \text { bilateral } \\
\quad \text { Permanent hearing loss that does not permit the child to understand directions of } \\
\quad \text { examiner and communicate despite amplification }\end{array}$ \\
\hline De Haan [65] & BSID-II-NL & $\begin{array}{l}\text { One or more of the following: } \\
\text { - } \quad \mathrm{CP} \pm \text { clinical hearing loss or visual handicaps } \\
\text { - } \quad \mathrm{MDI}<85 \text { or PDI }<85\end{array}$ \\
\hline Dilli [66] & BSID-II, GMFCS & $\begin{array}{ll}\text { One or more of the following: } \\
- & \text { Moderate-severe CP } \\
- & \text { MDI }<70, \text { PDI }<70 \\
- & \text { Bilateral deafness } \\
- & \text { Bilateral blindness }\end{array}$ \\
\hline Mitha [47] & Kaufman Assessment Battery for Children & $\begin{array}{l}\text { - } \quad \mathrm{CP} \text { (at least two of the following: abnormal posture or movement, increased } \\
\text { tone, hyperreflexia) } \\
\text { - } \mathrm{MPC}<70\end{array}$ \\
\hline Alshaikh [57] & BSID-II, WPPSI-Revised, Stanford-Binet IV & $\begin{array}{l}\text { One or more of the following: } \\
\text { - } \quad \mathrm{CP} \text { (non-progressive motor impairment characterised by abnormal muscle tone in at least } \\
\text { one extremity and decreased range or control of movements) } \\
\text { - } \quad \text { Cognitive delay }>2 \mathrm{SD} \text { below mean on standardised assessment (WPPSI-Revised, BSID-II } \\
\text { or Stanford-Binet IV) } \\
\text { - } \quad \text { Sensorineural hearing loss requiring amplification } \\
\text { - } \quad \text { Visual acuity }<20 / 200 \text { following refractive correction }\end{array}$ \\
\hline Yang [59] & $\begin{array}{l}\text { CBCL, “Current Status Survey”, WISC-IV, MINI-KID (for } \\
\text { ADHD, anxiety/mood disorders), DSM-IV-TR (for ASD) }\end{array}$ & $\begin{array}{l}\text { "Disabled" = when parents disclosed that the child received a handicap status as issued by the } \\
\text { Ministry of the Interior of Taiwan. Handicap is defined as disadvantaged condition, deriving } \\
\text { from impairment or disability limiting a person performing a role considered normal in respect } \\
\text { of their age, sex and social and cultural factors. }\end{array}$ \\
\hline
\end{tabular}


Table 2. Cont.

\begin{tabular}{|c|c|c|}
\hline Study & Scale Used & NDI Definitions \\
\hline Maruyama [60] & KPSD & $\begin{array}{l}\text { One or more of the following: } \\
\text { - } \quad \mathrm{CP} \\
\text { - } \quad \text { Unilateral or bilateral blindness } \\
\text { - } \quad \text { Severe hearing impairment } \\
\text { - } \quad \text { Developmental delay: } \mathrm{DQ}<70\end{array}$ \\
\hline Synnes [48] & BSID-III, GMFCS & \begin{tabular}{ll}
\multicolumn{2}{l}{ Severe NDI } \\
- & CP with GMFCS III, IV or V \\
- & Bayley-III motor composite $<70$, cognitive composite $<70$, language composite $<70$ \\
- & Hearing aid or cochlear implant \\
- & Bilateral visual impairment \\
NDI & \\
- & CP with GMFCS I \\
- & Bayley-III motor composite $<85$, cognitive $<85$, language composite $<85$ \\
- & Sensorineural/mixed hearing loss \\
- & Unilateral or bilateral visual impairment
\end{tabular} \\
\hline Bright [49] & $\begin{array}{l}\text { GMFCS, DAS-II, OWLS, NEPSY-II, WIAT-III, Manual Ability } \\
\text { Classification Test }\end{array}$ & $\begin{array}{l}\text { - } \quad \text { Parent-reported legally blind—severe visual impairment } \\
\text { - Severe auditory impairment—-parent-reported child has hearing aids or cochlear plant } \\
\text { and/or receives special services for the hearing-impaired }\end{array}$ \\
\hline \multirow{2}{*}{ Bolisetty [50] } & \multirow{2}{*}{ BSID-III, GMFCS } & $\begin{array}{l}\text { Moderate } \\
\text { - } \quad \text { BSID-III 2-3 SD below mean } \\
\text { - } \quad \text { Moderate CP GMFCS level } 2 \text { or } 3 \text { (able to walk with the assistance of aids) } \\
\text { - } \quad \begin{array}{l}\text { Bilateral deafness (requiring amplification with hearing aids or unilateral/bilateral } \\
\text { cochlear implants) }\end{array}\end{array}$ \\
\hline & & $\begin{array}{l}\text { Severe } \\
\text { - } \quad \text { BSID-III }>3 \text { SD below mean } \\
\text { - } \quad \text { Severe CP GMFCS level } 4 \text { or } 5 \text { (unable to walk with the assistance of aids) } \\
\text { - } \quad \text { Bilateral blindness (visual acuity of }<6 / 60 \text { in better eye) }\end{array}$ \\
\hline \multicolumn{3}{|c|}{ Studies Which Reported Continuous Outcomes } \\
\hline Shah [55] & BSID-II & $\mathrm{N} / \mathrm{A}$ \\
\hline Van der Ree [64] & $\begin{array}{c}\text { Bax' criteria, GMFCS, Movement ABC, WISC-III-NL, } \\
\text { NEPSY-II, AVLT, TEA-Ch, ADHD questionnaire, BRIEF, } \\
\text { CBCL }\end{array}$ & N/A \\
\hline
\end{tabular}


Table 2. Cont

\begin{tabular}{|c|c|c|}
\hline Study & Scale Used & NDI Definitions \\
\hline Hentges [58] & BSID-II & N/A \\
\hline Young [61] & $\begin{array}{c}\text { At } 2 \text { years: BSID-III } \\
\text { At } 4 \text { years: WPPI-III, CLEF-2, Beery-Buktenica Test of Visual } \\
\text { Motor Integration, the Behaviour Assessment System for } \\
\text { Children Parent Rating Scales, Behavioural Rating Inventory } \\
\text { of Executive Functioning-Preschool }\end{array}$ & N/A \\
\hline Zonnenberg [62] & BSID-II, Lexilijst (lexical development questionnaire), CBCL & $\mathrm{N} / \mathrm{A}$ \\
\hline \multicolumn{3}{|c|}{$\begin{array}{l}\text { Abbreviations: NDI-neurodevelopmental impairment; CP-cerebral palsy; BSID-II-Bayley Scales of Infant Development, Second Edition; MDI-Mental Development Index; } \\
\text { PDI-Psychomotor Development Index; KPSD-Kyoto Scale of Psychological Development; DQ-Development Quotient; GMFCS-Gross Motor Function Classification System; } \\
\text { Bayley-III-Bayley Scales of Infant and Toddler Development, Third Edition; MPC-Mental Processing Composite score; WPPSI-Revised-Wechsler Preschool and Primary Scales of } \\
\text { Intelligence, Revised; CBCL-Child Behaviour Checklist; WISC-IV-Wechsler Intelligence Scale for Children-IV; MINI-KID-Mini-International Neuropsychiatric Interview for Children } \\
\text { and Adolescents; DAS-II-School-Age Differential Ability Scales, Second Edition; OWLS-Oral Written Language Scales; NEPSY-II-Neuropsychological Assessment, Second Edition; } \\
\text { WIAT-III-Wechsler Individual Achievement Test, Third Edition; M-ABC-Movement Assessment Battery for Children; WISC-III-Wechsler Intelligence Scale for Children, Third } \\
\text { edition; AVLT-Rey's Auditory Verbal Learning Test; TEA-Ch-Test of Everyday Attention for Children; BRIEF-Behavior Rating Inventory of Executive Function; WPPSI-III-Wechsler } \\
\text { Preschool and Primary Scales of Intelligence, Third edition; CELF-2-Clinical Evaluation of Language Fundamentals-Preschool, Second Edition; WASI-Wechsler Abbreviated Scale of } \\
\text { Intelligence; M-ABC2-Movement Assessment Battery for Children, Second Edition; CELF-4-Clinical Evaluation of Language Fundamentals, Fourth Edition; CLI-Core Language Index; } \\
\text { WMTB-C-Working Memory Test Battery for Children; SD—-standard deviation(s); N/A—not applicable. }\end{array}$} \\
\hline
\end{tabular}




\subsection{Quantitative Synthesis}

Fourteen out of the 19 studies reporting dichotomised neurodevelopmental outcomes contained adequate information for meta-analysis. Table 3 details the number of NDI identified in the sepsis and non-sepsis groups in each study. Overall, $35 \%$ of the individuals with culture-proven sepsis had NDI compared with $17 \%$ in the non-sepsis group. The median attrition rate in these studies was $18 \%$ (range $3 \%-45 \%)$. Figure 3 demonstrates the OR forest plot based on available case analysis and a random effect model. Repeated analyses examining the impact of missing data demonstrated similar outcome trends. The forest plot demonstrated that VP infants with neonatal sepsis had more adverse long-term NDI compared with those without sepsis (OR 3.18, 95\% CI 2.29-4.41), as seen in Figure 3. Substantial heterogeneity existed across the studies $\left(\mathrm{I}^{2}=83.1,95 \%\right.$ CI $\left.73-89\right)$. The estimated predictive value was 1.02-9.89.

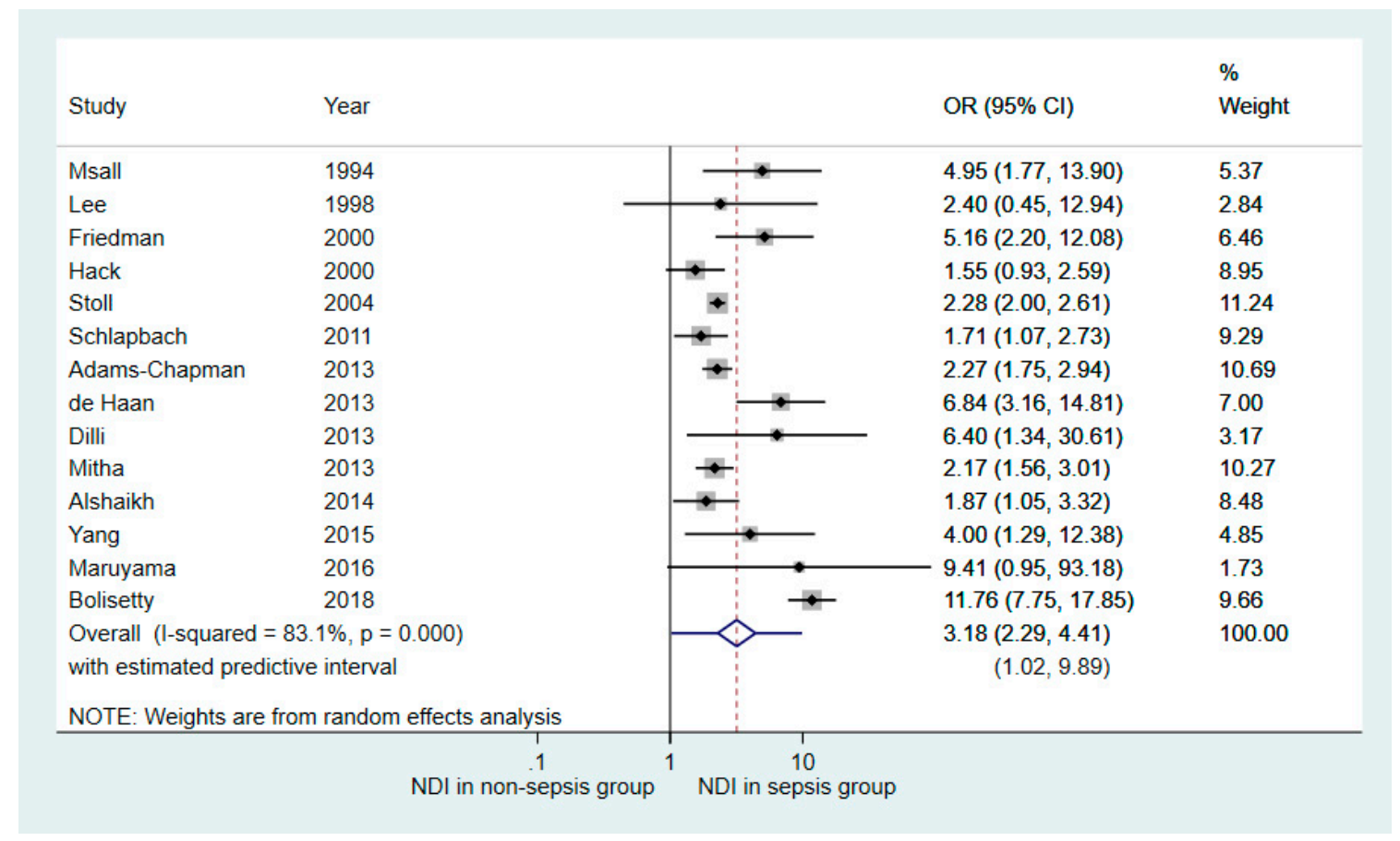

Figure 3. Forest plot showing the results of random effects meta-analysis of the 14 studies comparing neurodevelopmental outcomes in very premature infants with and without neonatal sepsis exposure (Outcome: number of participants with neurodevelopmental impairment). NDI—neurodevelopmental impairment; $\mathrm{OR}$-odds ratio; $\mathrm{CI}$ —confidence interval.

The contour-enhanced funnel plot shown in Figure 4 demonstrates a high likelihood of publication bias, as reflected by high degrees of plot asymmetry with a lack of negative studies. There was a predominance of studies showing statistically significant positive effect. However, on visual assessment of the plot, the publication bias was unlikely due to small study effects. The studies which demonstrated statistically significant findings (i.e., those lying outside the $1 \%$ line $(p<0.01))$ were not restricted to studies with small sample size. This was confirmed quantitatively using the Egger's meta-regression test. This test demonstrated that smaller studies did not give different results when compared with larger studies as the $95 \% \mathrm{CI}$ of the intercept did include the zero value (coefficient $1.33,95 \% \mathrm{CI}$ $-0.76-3.42, p=0.190)$. 
Table 3. Summary of the 19 studies with reported dichotomised neurodevelopmental outcomes.

\begin{tabular}{|c|c|c|c|c|}
\hline \multicolumn{5}{|c|}{ Number of Infants with NDI } \\
\hline Study & Sepsis Group & No Sepsis Group & Attrition Rate & Comments \\
\hline Msall [51] & $9 / 18(50 \%)$ & $22 / 131(17 \%)$ & $3 \%$ & $\mathrm{~N} / \mathrm{A}$ \\
\hline Lee [63] & $4 / 14(29 \%)$ & $3 / 21(14 \%)$ & N/A & N/A \\
\hline Friedman [52] & $11 / 27(41 \%)$ & $32 / 272(12 \%)$ & $10 \%$ & $\mathrm{~N} / \mathrm{A}$ \\
\hline Hack [53] & $43 / 93(46 \%)$ & $62 / 112(55 \%)$ & $8 \%$ & $\mathrm{~N} / \mathrm{A}$ \\
\hline Hoekstra [54] \# & NS & NS & $13 \%$ & $\begin{array}{c}\text { At } 47.5 \text { months (range } 36-60 \text { ), there was no statistically significant association between primary } \\
\text { or secondary sepsis and NDI. }\end{array}$ \\
\hline Stoll [43] & $861 / 1778(48 \%)$ & $576 / 1976(29 \%)$ & $20 \%$ & N/A \\
\hline Kono [44] \# & NS & NS & $36 \%$ & $\begin{array}{l}\text { At 36-42 months, there was an association between sepsis and cerebral palsy or death (OR 2.6, } \\
95 \% \text { CI 1.4-4.8) as well as sepsis and NDI or death (OR 2.8, 95\% CI 1.6-4.8). }\end{array}$ \\
\hline Jang [56] \# & NS & NS & N/A & $\begin{array}{l}\text { At } 18-24 \text { months, univariate analysis showed weak association between sepsis and cerebral } \\
\text { palsy (OR } 1.653,95 \% \text { CI } 0.849-3.215) .\end{array}$ \\
\hline Schlapbach [45] & $46 / 134(34 \%)$ & $55 / 235(23 \%)$ & $23 \%$ & N/A \\
\hline Adams-Chapman [46] & $148 / 474(31 \%)$ & $153 / 917(17 \%)$ & $16 \%$ & N/A \\
\hline De Haan [65] & $28 / 50(56 \%)$ & $16 / 102(17 \%)$ & $10 \%$ & N/A \\
\hline Dilli [66] & $8 / 13(62 \%)$ & $4 / 20(20 \%)$ & N/A & $\mathrm{N} / \mathrm{A}$ \\
\hline Mitha [47] & $84 / 643(14 \%)$ & $73 / 1126(6 \%)$ & $22 \%$ & N/A \\
\hline Alshaikh [57] & $26 / 105(25 \%)$ & $34 / 227(15 \%)$ & $13 \%$ & N/A \\
\hline Yang [59] & $13 / 26(50 \%)$ & $7 / 35(20 \%)$ & $45 \%$ & N/A \\
\hline Maruyama [60] & $3 / 4(75 \%)$ & $37 / 153(24 \%)$ & $28 \%$ & $\mathrm{~N} / \mathrm{A}$ \\
\hline Synnes [48] \# & NS & NS & $20 \%$ & $\begin{array}{l}\text { At 18-21 months, there was significant association between sepsis and significant NDI (OR 1.50, } \\
95 \% \text { CI 1.05-1.86), but no information was reported on association between sepsis and NDI. }\end{array}$ \\
\hline Bright [49] \# & NS & NS & $8 \%$ & $\begin{array}{l}\text { At } 10 \text { years of age, children who had confirmed bacteraemia were associated with lower } \\
\text { z-scores in verbal and nonverbal IQ, oral expression, academic achievement, executive function } \\
\text { and visual impairment. They were also more likely to have visual and auditory impairment but } \\
\text { not motor deficits. After adjusting for IQ, many of these associations were lost. }\end{array}$ \\
\hline Bolisetty [50] & $138 / 526(26 \%)$ & $29 / 988(3 \%)$ & $20 \%$ & N/A \\
\hline Total & $1422 / 3905(36 \%)$ & $1103 / 6315(17 \%)$ & Median: $18 \%$ & \\
\hline
\end{tabular}

\#: studies did not report absolute numbers for NDI for each study group. NS-not specified; N/A—not applicable. 


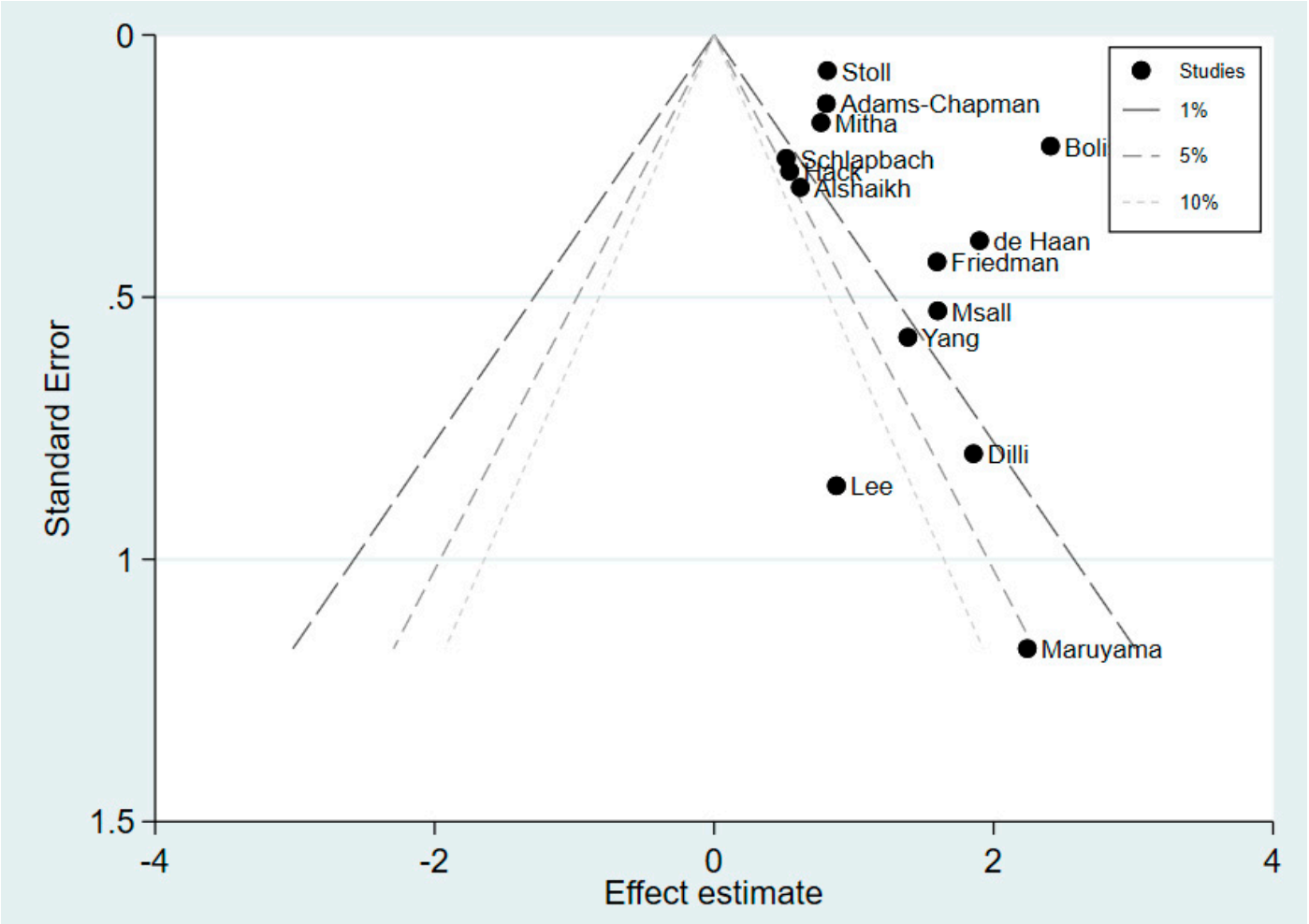

Figure 4. Contour-enhanced funnel plot of the 14 studies with reported dichotomised neurodevelopmental outcomes.

Results of subanalysis based on the four studies with a follow-up duration of 36 months or greater showed similar association between neonatal sepsis and NDI (OR 3.07, 95\% CI 1.79-5.28; Figure 5), as compared to the primary meta-analysis conducted from all 14 studies (OR 3.18, 95\% CI 2.29-4.41), as seen in Figure 3.

\begin{tabular}{|c|c|c|c|c|}
\hline \multirow[b]{2}{*}{ Study } & \multirow{2}{*}{\multicolumn{2}{|c|}{ Year }} & \multirow[b]{2}{*}{ OR $(95 \% \mathrm{Cl})$} & \multirow{2}{*}{$\begin{array}{l}\% \\
\text { Weight }\end{array}$} \\
\hline & & & & \\
\hline Msall & 1994 & - & $4.95(1.77,13.90)$ & 20.02 \\
\hline Mitha & 2013 & + & $2.17(1.56,3.01)$ & 57.32 \\
\hline Yang & 2015 & - & $4.00(1.29,12.38)$ & 17.48 \\
\hline Maruyama & 2016 & $\longrightarrow$ & $9.41(0.95,93.18)$ & 5.19 \\
\hline Overall (I-s & $d=31.1 \%, p=0.226)$ & & $3.07(1.79,5.28)$ & 100.00 \\
\hline with estima & edictive interval & & $(0.49,19.21)$ & \\
\hline \multicolumn{2}{|c|}{ NOTE: Weights are from random effects analysis } & & & \\
\hline & $\mathrm{NDI}$ in non-sepsis group ${ }^{1}$ & $\begin{array}{c}10 \\
N D I\end{array}$ & & \\
\hline
\end{tabular}

Figure 5. Forest plot of the subanalysis of studies which had a follow-up duration of 36 months or greater. The plot shows the results of random effects meta-analysis of the four studies comparing neurodevelopmental outcomes in very premature infants with and without neonatal sepsis exposure (Outcome: number of participants with neurodevelopmental impairment). 


\subsection{Qualitative Synthesis}

The qualitative synthesis included five studies which reported dichotomised outcomes $[44,48,49,54,56]$ but did not have adequate information for meta-analysis, and five studies which reported continuous outcomes [55,58,61,62,64].

Table 3 summarises the five studies which reported dichotomised outcomes. They revealed conflicting findings, as some studies demonstrated no or minimal association between sepsis and NDI [54,56], whilst others showed an association between sepsis and NDI [44,48]. One study showed an association between sepsis and NDI which they argued was attributable to the effect of IQ [49]. Table 4 summarises the five studies which reported continuous outcomes [55,58,61,62,64]. Four of the studies demonstrated no or minimal association $[55,58,61,62,64]$ and only one found significant association between sepsis and cognitive function in the studied VP children [62].

Table 4. Summary of the five studies with reported continuous neurodevelopmental outcomes.

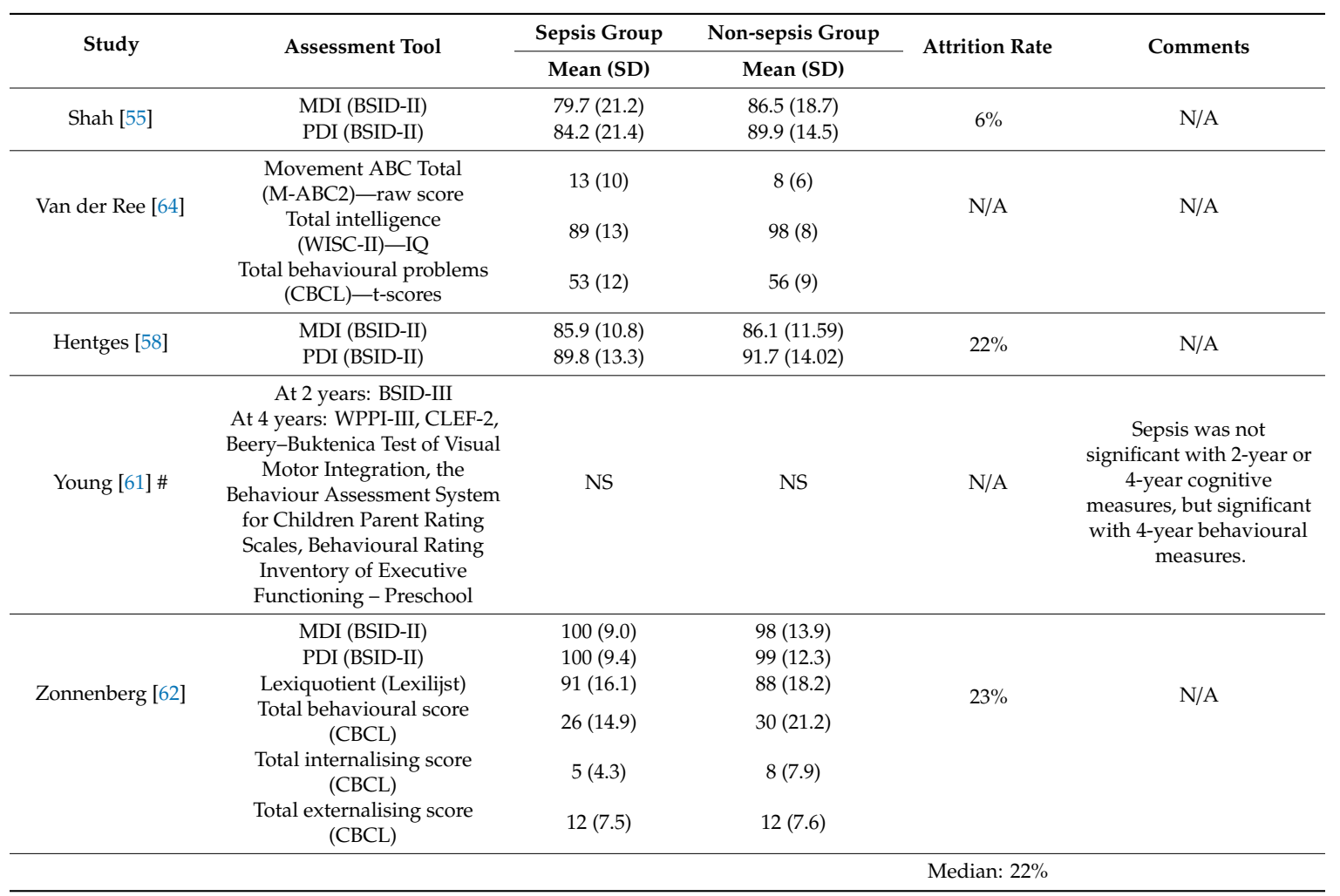

\#: Studies did not report group mean and SD for sepsis and non-sepsis group. NS-not specified.

The following sections provide detailed accounts of each study included in this qualitative synthesis.

\subsubsection{Studies Reporting Dichotomised Outcomes}

Hoekstra et al. [54] retrospectively analysed results from 675 out of 778 ( $87 \%$ follow-up rate) children born at 23-26 weeks gestational age and assessed them between 36 and 60 months of age. The association between sepsis and NDI at a mean age of 47.5 months (range 36-60 months) was not statistically significant. No detailed psychometric testing was done at school age.

Jang et al. [56] retrospectively reviewed medical records of VLBW infants admitted from 1998 to 2007 and compared with those admitted from 1989 to 1997 . There was a total of 967 survivors. Univariate analysis showed sepsis was a weak risk factor for cerebral palsy (OR 1.653, 95\% CI $0.849-3.215)$ assessed at $18-24$ months. No information was reported on sepsis and noncerebral palsy NDI. 
Kono et al. [44] evaluated a prospective cohort of 2847 VLBW survivors. A total of 1826 (64\%) completed follow-up at 36-42 months of age. Of the 1826, 113 survivors had neonatal sepsis. An association was found between sepsis and a combined measure of cerebral palsy or death (OR 2.6, 95\% CI 1.4-4.8) as well as sepsis and a combined measure of NDI or death (OR 2.8, 95\% CI 1.6-4.8). No information was reported between sepsis and NDI only.

Synnes et al. [48] evaluated a prospective national cohort of children born at less than 29 weeks gestational age at 18-21 months. Eighty percent (1870 out of 2340) of the infants completed follow-up. Outcomes were differentiated into NDI and significant NDI based on a prespecified criterion, as seen in Table 2. They found a statistically significant association between sepsis and significant NDI (OR $1.50,95 \%$ CI 1.05-1.86), but no information was reported on association between sepsis and NDI.

Bright et al. [49] reviewed a multicentre prospective study of extremely preterm infants born before 28 weeks gestational age. Ninety-two percent of infants were followed up (889 out of 966) with cognitive assessments at ten years of age, of which 233 infants had neonatal bacteraemia. A wide variety of outcomes were assessed. Confirmed bacteraemia was associated with lower z-scores in outcomes assessing verbal and nonverbal IQ, oral expression, academic achievement, executive function and visual perception. Children with confirmed bacteraemia were more likely to have visual or auditory impairment, but not motor impairment. However, after adjusting for IQ, many of these associations were lost, suggesting IQ may be a mediator between bacteraemia and cognitive deficits.

\subsubsection{Studies Reporting Continuous Outcomes}

Shah et al. [55], Hentges et al. [58] and Zonnenberg et al. [62] assessed mental development index (MDI) and psychomotor development index (PDI) outcomes of VP infants at 24 months. Shah et al. [55] reported an unadjusted mean difference of 6.8 (95\% CI 0.8-12.8, $p=0.02)$ in MDI and $5.6(95 \% \mathrm{CI}$ $0.4-10.9, p=0.04$ ) in PDI between VP infants with and without sepsis. After adjusting for potential confounders and white matter abnormalities, group differences decreased and there was no statistical significance, suggesting white matter abnormalities may mediate functional impairment. Hentges et al. [58] and Zonnenberg et al. [62] reported no significant mean difference in MDI and PDI between the two study populations. Zonnenberg et al. [62] found no significant difference in lexical development or behaviour scores between the two groups.

Young et al. [61] reviewed 100 infants born before 32 weeks gestational age at 24 months and 48 months. Using bootstrap ratios of predictors and partial least square regression to draw relationships, they found sepsis was not significantly associated with 2-year or 4-year cognitive measures but was significantly associated with 4-year behavioural measures.

Van der Ree et al. [64] evaluated 50 VP infants (of which 32 had neonatal sepsis) at 6-9 years of age using an extensive list of motor, cognitive and behavioural outcomes. The study demonstrated $68 \%$ of the proven sepsis cohort had abnormal or borderline motor skills (OR 3.30, 95\% CI 0.98-11.07, $p$ $<0.10$ ), particularly poorer fine motor skills (OR 5.46, 95\% CI 1.52-1.58, $p<0.01$ ). Their total IQ was 89 (14 SD, range 55-118) compared with 98 (8 SD, range 82-110) in VP infants without sepsis, giving an OR of 13.22 (95\% CI 1.57-111.75, $p<0.05)$. Verbal memory and attention were significantly affected in those with proven sepsis compared with VP infants without sepsis $(0.61 \mathrm{SD}, 95 \%$ CI 0.04-1.17, $p=$ 0.033 and 0.94 SD, 95\% CI 0.32-1.62, $p=0.011$, respectively). There were no significant differences in total behavioural problems between the groups (OR 0.86, 95\% CI 0.25-3.00).

\section{Discussion}

This review provides an updated literature synthesis on the long-term neurodevelopmental impact of neonatal sepsis in VP infants compared to those without sepsis. The conducted meta-analysis demonstrated statistically significant association between sepsis and NDI, but was limited by the substantial heterogeneity that existed between studies. On the contrary, qualitative synthesis of the remaining studies revealed less clear associations between sepsis and long-term neurocognitive deficits. 
There were limited studies examining continuous outcomes of NDI after sepsis. The included studies in this review offer valuable insight, but will need to be interpreted in the context of the study limitations.

\subsection{Study Designs}

All studies were observational studies, as randomised control trials were not ethically possible given the nature of the topic. In case-control studies, controls were generally matched for admission period, gestational age and/or birth weight. These studies may have suffered from selection bias and reporting bias. One case-control study reported they selected controls by randomly selecting every fifth subject admitted to the NICU within a specific time-frame [65]. Case-cohort studies used either retrospectively collected data or prospectively collected data. Prospective data collection is dependent on the proportion of infants successfully followed up which determines attrition bias. Retrospective data collection can be limited as not all information for the study may be collected at the time, which can result in reporting bias. In addition, all retrospective reviews rely on accurate data entries and recordings.

In retrospective studies, increasing sample size results in increasing statistical power. Some studies included in this review had much smaller study populations compared with other studies (i.e., the sample size in all included studies ranged from 33 to 7892). This would contribute to the overall statistical heterogeneity and impede the validity and interpretation of study outcome. Adams-Chapman et al. [46] reported that the non-sepsis group in the Candida study was not representative of the homogeneous extremely low birthweight population and thus performed a secondary analysis using additional non-sepsis infants enrolled in the registry by the Neonatal Research Network (NRN) of the Eunice Kennedy Shriver National Institute of Child Health and Health Development (NICHD). Although this increases statistical power, it also increases the risk of selection and reporting bias.

Similarly, several of the included studies consisted of disproportionate sample size in the sepsis versus the non-sepsis group, with up to ten times greater numbers in the non-sepsis group compared to the sepsis group in one study [51,52]. For example, Msall et al. [51] investigated sepsis as a perinatal factor and of the 149 participants followed up, only 18 had confirmed sepsis. Van der Ree et al. [64] compared 32 infants with sepsis to 18 non-sepsis infants. Due to the disparity in group numbers, with much smaller numbers in the sepsis group, there is a higher chance of over-representing infants developing NDI in the sepsis group as compared to the much larger non-sepsis group. This overestimation can result in publication bias as studies are more inclined to publish results with significant findings.

\subsection{Population}

All studies adjusted for selected confounding factors such as age and sex. However, they adjusted differently with various combinations of other factors such as multiple birth, mode of delivery, corticosteroid exposure, intrauterine growth restriction, bronchopulmonary dysplasia, necrotising enterocolitis, chorioamnionitis, periventricular leukomalacia, socioeconomic status and maternal education. As a result, we extracted unadjusted numerical data to pool, but were unable to assess the impact of these confounding factors.

The median attrition rate was $20 \%$ and the range was wide $(3 \%-45 \%)$. Loss through follow-up can be due to many factors, such as unable to be contacted, parent refusal, or being too impaired to participate in tests [67]. Most studies did not report the reason for loss to follow-up. Some studies compared patient demographics between infants who completed follow-up with those who did not to identify attrition bias. As loss to follow-up increases, bias in the reported outcome also increases [68]. Prevention of attrition bias is inherently difficult and best to be addressed in prospectively designed studies.

One potential confounder may be related to the wide range in birth years of the infants, and different ages at assessment. For instance, infants born in earlier eras may have received less advanced neonatal care as compared to more recent eras. Furthermore, quality of neonatal care may vary across 
regions and countries, potentially influencing the events experienced by the infant during the neonatal period and the subsequent neurodevelopmental outcomes.

\subsection{Assessment of Sepsis}

One limitation of this review was the definition of neonatal sepsis as an appreciable portion of neonatal sepsis is blood culture-negative or have positive cultures from other sterile sites. For a focused review, we defined sepsis as having a blood culture-proven infection which has historically been the 'gold standard', but there was great variability amongst studies [4,69]. Definitions included: positive blood culture only $[44,46,48,49,52,54,56,59,60,62-64,66]$; positive blood culture and clinical signs of sepsis [50,53,58,65]; positive blood culture and antibiotic use [43,47,51,61]; positive blood culture, clinical signs of sepsis and antibiotic use [57]; positive blood culture, antibiotic use and presence of raised inflammatory markers [55]; and positive blood culture, clinical signs of sepsis, antibiotic use and presence of raised inflammatory markers [45]. Other specific variations include time that the blood culture was taken, duration of antibiotic use and type of infection laboratory markers examined. Some of these studies justified that using a blood culture only definition has suboptimal sensitivity and specificity, as there is risk of contamination which could produce false positives [55]. Infants who had clinically suspicious sepsis, but no positive blood culture may also be missed. The diversity in sepsis definitions could contribute to the population heterogeneity and overall selection bias.

Selected studies that analysed the impact of specific micro-organisms may also contribute to selection bias. Lee et al. [63], Friedman et al. [52], and Adams-Chapman et al. [46] focused on Candidaemia, De Haan et al. [65] examined Candidaemia and Gram-negative organisms, whereas Alshaikh et al. [57] investigated coagulase-negative Staphylocci specifically. In contrast, Stoll et al. [43], Schlapbach et al. [45], and Hentges et al. [58] did a micro-organism subtype analysis and reported the association between predominant organisms and outcomes. For instance, Hentges et al. [58] found no statistical difference between the two study groups, but upon further analysis, reported VLBW infants with Gram-positive sepsis showed higher rates of motor deficit when compared to the non-sepsis group (68.8\% and $29.3 \%$ respectively). Similarly, Schlapbech et al. [45] did a subtype analysis and found infants who had Gram-positive sepsis had the poorest outcomes. This suggests different organisms may cause different deficits which should be further explored in future studies.

\subsection{Assessment of Outcomes}

Functional outcomes must be interpreted carefully given the variability between studies. Most studies focused on early outcomes, with half of the included studies in this review performing assessments at or before the age of 24 months. Only five studies examined outcomes beyond 5 years of age $[47,49,51,59,64]$, two of which investigated outcomes beyond the age of ten $[49,59]$. No studies assessed outcomes in early adulthood or reported if difficulties persisted into early adulthood. Cognitive skills are complex and may evolve overtime with brain maturation. Early assessments may underestimate the full spectrum of outcomes, such as milder motor dysfunctions and specific learning disorders, and be of less predictive value for long-term outcomes $[45,66]$. Most studies performed a single cross-sectional follow-up, but outcomes may alter over time. Children who test within the normal range at early follow-up may still remain at risk of developing significant problems later in life $[43,54,57]$. Conversely, children with early deficits may improve or remain unchanged.

Eighteen out of the 24 included studies did not specify if outcome assessors were blinded to the infants' neonatal history $[43-46,48,50,52,53,55,56,59-61,63-66]$. Five studies reported blinding of outcome assessors $[49,51,57,58,62]$ and one reported no blinding was performed [54]. Without blinding, there is a high chance of detection and reporting bias as assessors may perceive sepsis-affected infants to be at higher risk of functional deficits. Although the tests are standardised, most studies did not note the experience level of outcome assessors, which may also contribute to the risk of detection and reporting bias. 
Two approaches were used to assess outcomes: using dichotomised (using a prespecified NDI definition) or continuous (calculating mean and SD of groups) outcomes. In the former, variation in NDI definition was a significant limitation. Most studies defined NDI as cerebral palsy or its components, cognitive and psychomotor scores of $<70$ or more than two SD below the mean, visual impairment or hearing impairment. Lee et al. [63] defined cognitive delay as more than three SD below the mean which would exclude children who scored between two and three SD below the mean. In contrast, Yang et al. [59] utilised a different definition whereby the parent had to disclose that the child was 'disabled', a handicap status issued by the Ministry of the Interior of Taiwan, which is a subjective evaluation of disability. Fifteen studies used BSID-II as an assessment tool where MDI and PDI was defined as a score of $<70$ or more than two SD below the mean. BSID-II is useful for infants who are yet to enter preschool as it provides an indicator if the infant is at risk of developmental delay, therefore the caregiver has the opportunity to seek early intervention to improve outcomes. The 2005 BSID revision saw a shift from BSID-II to Bayley-III. Four studies used Bayley-III for assessment [46,48,50,61]. However, studies have reported that the Bayley-III detects lower rates of NDI in infants as compared to BSID-II using the same population [70-72]. There is also limited data on the long-term predictive validity of Bayley-III [72]. Kono et al. [44], De Haan et al. [65] and Maruyama et al. [60] looked at NDI and death together, which may overestimate the study effect of sepsis in VP infants. Overall, dichotomised studies tended to focus on identifying the most impaired children, often at an early age. This was reflected by the reporting of incomplete assessments in several studies due to reasons such as distractions or limitations in the child's ability to complete the assessment.

Accordingly, less focus was placed on less impaired children who may have more subtle cognitive difficulties. To address this, studies using continuous outcomes can identify subtle impairments as a child grows older and begins school. For instance, Van der Ree et al. [64] noted that out of the 18 children with neonatal sepsis exposure who had normal full-scale IQs, nine of them had problems in attention or memory. Limited studies $(n=5)$ reported continuous outcome variables, and the heterogeneity of the reporting cognitive domains in each study made it difficult to pool these results. In these studies, the mean results were comparable between the sepsis group and non-sepsis group. One potential factor contributing to the conflicting results may be the Zonnenberg et al. [62] study, as they compared proven sepsis versus no proven sepsis in a cohort that all had an episode of suspicious infection, which questions the incorporation of clinically suspicious infants into our analysis and introduces selection bias. Only one study used a wide variety of assessments in a continuous manner to extensively study the neurocognitive deficits a child may be experiencing [64].

\subsection{Review Limitations}

This review has several limitations. Data screening, extraction, and analysis were performed by one reviewer (S.C.), instead of consensus reached by at least two independent reviewers. However, data synthesis was discussed with, and consensus was reached with the senior author (J.Y.-M.Y). The reviewer did not attempt to contact the included study authors for missing data. Thus, this systematic review did not strictly adhere to the recommended Preferred Reporting Items for Systematic Reviews and Meta-analyses (PRISMA) guidelines [73]. Secondly, sensitivity analyses were not performed to examine the influence of attrition bias and the contribution of different micro-organisms. Given that substantial disagreement remains in the definition of sepsis, selecting a sepsis definition also subjects our review to this methodological limitation. Our review did not address the impact of neonatal meningitis and urinary tract infection separately, which are both common in the NICU setting.

\subsection{Recommendations for Future Research}

Based on the results of this systematic review, we recommend future studies examining cognitive outcomes of VP infants with neonatal sepsis. Longitudinal studies of prospectively collected data would be the most appropriate study design. Larger cohorts and high rates of follow-up would increase statistical power and reduce attrition bias. If follow-up rates are low, assessment of the characteristics 
between infants who do complete follow-up and those who do not would be needed. There is much evidence to support the risk of major NDI, but little research has been done in identifying more subtle difficulties in less impaired children which may be significant during activities of daily living. To dissect the specific cognitive impairments, a range of assessment tools should be used to measure motor function, cognitive skills and behaviour as continuous variables. Participants should be assessed later in life, ideally once they have entered the education curriculum, as there is a higher predictive value in dissecting long-term outcomes.

\section{Conclusions}

Neonatal sepsis can have a profound impact on neurodevelopment of VP infants. This systematic review found 24 published studies and performed meta-analysis in 14 studies using prespecified NDI definitions. This review suggests that the most impaired VP infants surviving neonatal sepsis may be at higher risk for long-term neurodevelopmental disability compared with VP infants without sepsis. However, current evidence is limited by significant statistical heterogeneity and publication bias due to significant differences in the included study design and definitions used for NDI. Moreover, this finding could not be extrapolated to all VP infants surviving neonatal sepsis due to lack of studies reporting cognitive outcomes using non-discrete, continuous variables. Current published studies lack long-term longitudinal follow-up data. These results highlight the necessity for future longitudinal studies to use continuous outcomes performed at a later age to discern the subtle and more specific long-term cognitive risks for VP children with neonatal sepsis.

Supplementary Materials: The following are available online at http://www.mdpi.com/2227-9067/6/12/131/s1, Table S1. Detailed literature search strategy, Table S2. The Cochrane Collaboration's tool for assessing risk of bias, Table S3. Assessment of risk of bias of the 24 included studies.

Author Contributions: Conceptualization, S.C., D.K.T., P.J.A. and J.Y.-M.Y.; Methodology, S.C. and J.Y.-M.Y.; Formal Analysis, S.C. and J.Y.-M.Y.; Investigation, S.C., D.K.T., P.J.A. and J.Y.-M.Y.; Resources, D.K.T., P.J.A. and J.Y.-M.Y.; Writing-Original Draft Preparation, S.C.; Writing-Review \& Editing, S.C., D.K.T., P.J.A. and J.Y.-M.Y.; Supervision, D.K.T., P.J.A. and J.Y.-M.Y.; Project Administration, J.Y.-M.Y.; Funding Acquisition, D.K.T., P.J.A. and J.Y.-M.Y.

Funding: This project was financially supported by the Australian National Health and Medical Research Council (NHMRC) (Centre of Research Excellence Grant ID 1060733 and 1153176; Senior Research Fellowship ID 1081288 to P.J.A.; Career Development Fellowship ID 1160003 to D.K.T.), the Royal Children's Hospital Foundation (RCH 1000 to J.Y.-M.Y.), Murdoch Children's Research Institute, The University of Melbourne, Department of Paediatrics, and the Victorian Government's Operational Infrastructure Support Program.

Acknowledgments: We gratefully acknowledge support provided by the members of the Victorian Infant Brain Study (VIBeS), Developmental Imaging and Neuroscience Research groups, located at the Murdoch Children's Research Institute, Melbourne, Victoria. We also thank Poh Chua at the Royal Children's Hospital library for her advice and assistance in performing the literature search.

Conflicts of Interest: The authors have no conflict of interest to declare.

\section{References}

1. Shane, A.L.; Sanchez, P.J.; Stoll, B.J. Neonatal sepsis. Lancet 2017, 390, 1770-1780. [CrossRef]

2. Shah, B.A.; Padbury, J.F. Neonatal sepsis: An old problem with new insights. Virulence 2014, 5, 170-178. [CrossRef] [PubMed]

3. McCormick, M.C. The contribution of low birth weight to infant mortality and childhood morbidity. N. Engl. J. Med. 1985, 312, 82-90. [CrossRef] [PubMed]

4. Wynn, J.L. Defining neonatal sepsis. Curr. Opin. Pediatr. 2016, 28, 135-140. [CrossRef]

5. Boghossian, N.S.; Page, G.P.; Bell, E.F.; Stoll, B.J.; Murray, J.C.; Cotten, C.M.; Shankaran, S.; Walsh, M.C.; Laptook, A.R.; Newman, N.S.; et al. Late-onset sepsis in very low birth weight infants from singleton and multiple-gestation births. J. Pediatr. 2013, 162, 1120-1124. [CrossRef] [PubMed] 
6. Regev, R.H.; Arnon, S.; Litmanovitz, I.; Bauer-Rusek, S.; Boyko, V.; Lerner-Geva, L.; Reichman, B.; Israel Neonatal, N. Association between neonatal morbidities and head growth from birth until discharge in very-low-birthweight infants born preterm: A population-based study. Dev. Med. Child Neurol. 2016, 58, 1159-1166. [CrossRef]

7. Adams-Chapman, I.; Stoll, B.J. Neonatal infection and long-term neurodevelopmental outcome in the preterm infant. Curr. Opin. Infect. Dis. 2006, 19, 290-297. [CrossRef]

8. Khwaja, O.; Volpe, J.J. Pathogenesis of cerebral white matter injury of prematurity. Arch. Dis. Child. Fetal Neonatal Ed. 2008, 93, F153-F161. [CrossRef]

9. Volpe, J.J. Postnatal sepsis, necrotizing entercolitis, and the critical role of systemic inflammation in white matter injury in premature infants. J. Pediatr. 2008, 153, 160-163. [CrossRef]

10. Alshaikh, B.; Yusuf, K.; Sauve, R. Neurodevelopmental outcomes of very low birth weight infants with neonatal sepsis: Systematic review and meta-analysis. J. Perinatol. 2013, 33, 558-564. [CrossRef]

11. Cochrane Handbook for Systematic Reviews of Interventions. Available online: www.handbook.cochrane.org (accessed on 10 November 2019).

12. DerSimonian, R.; Laird, N. Meta-analysis in clinical trials. Control. Clin. Trials 1986, 7, 177-188. [CrossRef]

13. Kontopantelis, E.; Reeves, D. metaan: Random-effects meta-analysis. Stata J. 2010, 10, 395-407. [CrossRef]

14. Chaimani, A.; Mavridis, D.; Salanti, G. A hands-on practical tutorial on performing meta-analysis with Stata. Evid. Based Ment. Health 2014, 17, 111-116. [CrossRef] [PubMed]

15. Mantel, N.; Haenszel, W. Statistical aspects of the analysis of data from retrospective studies of disease. J. Natl. Cancer Inst. 1959, 22, 719-748.

16. Higgins, J.P.; Thompson, S.G.; Deeks, J.J.; Altman, D.G. Measuring inconsistency in meta-analyses. Br. Med. J. 2003, 327, 557-560. [CrossRef]

17. Riley, R.D.; Higgins, J.P.; Deeks, J.J. Interpretation of random effects meta-analyses. Br. Med. J. 2011, 342, d549. [CrossRef]

18. Peters, J.L.; Sutton, A.J.; Jones, D.R.; Abrams, K.R.; Rushton, L. Contour-enhanced meta-analysis funnel plots help distinguish publication bias from other causes of asymmetry. J. Clin. Epidemiol. 2008, 61, 991-996. [CrossRef]

19. Egger, M.; Davey Smith, G.; Schneider, M.; Minder, C. Bias in meta-analysis detected by a simple, graphical test. Br. Med. J. 1997, 315, 629-634. [CrossRef]

20. Paul, V.K.; Radhika, S.; Deorari, A.K.; Singh, M. Neurodevelopmental outcome of 'at risk' nursery graduates. Indian J. Pediatr. 1998, 65, 857-862. [CrossRef]

21. Wheater, M.; Rennie, J.M. Perinatal infection is an important risk factor for cerebral palsy in very-low-birthweight infants. Dev. Med. Child Neurol. 2000, 42, 364-367. [CrossRef]

22. McGrath, M.; Sullivan, M. Birth weight, neonatal morbidities, and school age outcomes in full-term and preterm infants. Issues Compr. Pediatr. Nurs. 2002, 25, 231-254. [CrossRef] [PubMed]

23. Benjamin, D.K., Jr.; Stoll, B.J.; Fanaroff, A.A.; McDonald, S.A.; Oh, W.; Higgins, R.D.; Duara, S.; Poole, K.; Laptook, A.; Goldberg, R.; et al. Neonatal candidiasis among extremely low birth weight infants: Risk factors, mortality rates, and neurodevelopmental outcomes at 18 to 22 months. Pediatrics 2006, 117, 84-92. [CrossRef] [PubMed]

24. Addison, K.; Griffin, M.P.; Moorman, J.R.; Lake, D.E.; O'Shea, T.M. Heart rate characteristics and neurodevelopmental outcome in very low birth weight infants. J. Perinatol. 2009, 29, 750-756. [CrossRef] [PubMed]

25. Kiechl-Kohlendorfer, U.; Ralser, E.; Pupp Peglow, U.; Reiter, G.; Trawoger, R. Adverse neurodevelopmental outcome in preterm infants: Risk factor profiles for different gestational ages. Acta Paediatr. 2009, 98, 792-796. [CrossRef]

26. Mercier, C.E.; Dunn, M.S.; Ferrelli, K.R.; Howard, D.B.; Soll, R.F. Neurodevelopmental outcome of extremely low birth weight infants from the vermont oxford network: 1998-2003 and the Vermont Oxford Network ELBW infant follow-up study group. Neonatology 2010, 97, 329-338. [CrossRef]

27. Gocer, C.; Kavuncuoglu, S.; Arslan, G.; Ertem, L.; Ozbek, S.; Ozturegen, E.; Akin, M.A.; Baysoy, N. Neurodevelopmental problems of very low birth weight premature infants and factors affecting neurological morbidity. Turk. Arch. Pediatr. 2011, 46, 207-214. [CrossRef] 
28. Schlapbach, L.J.; Adams, M.; Proietti, E.; Aebischer, M.; Grunt, S.; Borradori-Tolsa, C.; Bickle-Graz, M.; Bucher, H.U.; Latal, B.; Natalucci, G. Outcome at two years of age in a Swiss national cohort of extremely preterm infants born between 2000 and 2008. BMC Pediatr. 2012, 12. [CrossRef]

29. Barton, M.; O’Brien, K.; Robinson, J.L.; Davies, D.H.; Simpson, K.; Asztalos, E.; Langley, J.M.; Le Saux, N.; Sauve, R.; Synnes, A.; et al. Invasive candidiasis in low birth weight preterm infants: Risk factors, clinical course and outcome in a prospective multicenter study of cases and their matched controls. BMC Infect. Dis. 2014, 14, 327. [CrossRef]

30. Fairchild, K.D.; Sinkin, R.A.; Davalian, F.; Blackman, A.E.; Swanson, J.R.; Matsumoto, J.A.; Lake, D.E.; Moorman, J.R.; Blackman, J.A. Abnormal heart rate characteristics are associated with abnormal neuroimaging and outcomes in extremely low birth weight infants. J. Perinatol. 2014, 34, 375-379. [CrossRef]

31. Ferreira, R.C.; Mello, R.R.; Silva, K.S. Neonatal sepsis as a risk factor for neurodevelopmental changes in preterm infants with very low birth weight. J. Pediatr. (Rio J.) 2014, 90, 293-299. [CrossRef]

32. Chattopadhyay, N.; Mitra, K. Neurodevelopmental outcome of high risk newborns discharged from special care baby units in a rural district in India. J. Public Health Res. 2015, 4, 7-12. [CrossRef] [PubMed]

33. Picciolini, O.; Montirosso, R.; Porro, M.; Gianni, M.L.; Mosca, F. Neurofunctional assessment at term equivalent age can predict 3-year neurodevelopmental outcomes in very low birth weight infants. Acta Paediatr. 2016, 105, e47-e53. [CrossRef] [PubMed]

34. Shany, E.; Inder, T.E.; Goshen, S.; Lee, I.; Neil, J.J.; Smyser, C.D.; Doyle, L.W.; Anderson, P.J.; Shimony, J.S. Diffusion Tensor Tractography of the Cerebellar Peduncles in Prematurely Born 7-Year-Old Children. Cerebellum 2017, 16, 314-325. [CrossRef]

35. Abolfotouh, M.A.; Al Saif, S.; Altwaijri, W.A.; Al Rowaily, M.A. Prospective study of early and late outcomes of extremely low birthweight in Central Saudi Arabia. BMC Pediatr. 2018, 18. [CrossRef] [PubMed]

36. Adams-Chapman, I.; Heyne, R.J.; DeMauro, S.B.; Duncan, A.F.; Hintz, S.R.; Pappas, A.; Vohr, B.R.; McDonald, S.A.; Das, A.; Newman, J.E.; et al. Neurodevelopmental Impairment Among Extremely Preterm Infants in the Neonatal Research Network. Pediatrics 2018, 141. [CrossRef] [PubMed]

37. Chang, J.H.; Hsu, C.H.; Tsou, K.I.; Jim, W.T. Outcomes and related factors in a cohort of infants born in Taiwan over a period of five years (2007-2011) with borderline viability. J. Formos. Med. Assoc. 2018, 117, 365-373. [CrossRef] [PubMed]

38. Pawar, S.J.; Oleti, T.; Bharathi, S.; Tipparaju, S.; Mustafa, E. Growth and Neurodevelopmental Outcome in Preterm LBW Infants with Sepsis in India: A Prospective Cohort. Int. J. Pediatr. 2018, 2018, 5735632. [CrossRef]

39. Savioli, K.; Rouse, C.; Susi, A.; Gorman, G.; Hisle-Gorman, E. Suspected or known neonatal sepsis and neurodevelopmental delay by 5 years. J. Perinatol. 2018, 38, 1573-1580. [CrossRef]

40. Singh, L.; Das, S.; Bhat, V.B.; Plakkal, N. Early Neurodevelopmental Outcome of Very Low Birthweight Neonates with Culture-positive Blood Stream Infection: A Prospective Cohort Study. Cureus 2018, 10, e3492. [CrossRef]

41. Smilga, A.S.; Garfinkle, J.; Ng, P.; Andersen, J.; Buckley, D.; Fehlings, D.; Kirton, A.; Wood, E.; van Rensburg, E.; Shevell, M.; et al. Neonatal Infection in Children With Cerebral Palsy: A Registry-Based Cohort Study. Pediatr. Neurol. 2018, 80, 77-83. [CrossRef]

42. Rand, K.M.; Austin, N.C.; Inder, T.E.; Bora, S.; Woodward, L.J. Neonatal Infection and Later Neurodevelopmental Risk in the Very Preterm Infant. J. Pediatr. 2016, 170, 97-104. [CrossRef] [PubMed]

43. Stoll, B.J.; Hansen, N.I.; Adams-Chapman, I.; Fanaroff, A.A.; Hintz, S.R.; Vohr, B.; Higgins, R.D.; National Institute of Child Health; Human Development Neonatal Research Network. Neurodevelopmental and growth impairment among extremely low-birth-weight infants with neonatal infection. J. Am. Med. Assoc. 2004, 292, 2357-2365. [CrossRef] [PubMed]

44. Kono, Y.; Mishina, J.; Yonemoto, N.; Kusuda, S.; Fujimura, M.; Nicu Network, J. Neonatal correlates of adverse outcomes in very low-birthweight infants in the NICU Network. Pediatr. Int. 2011, 53, 930-935. [CrossRef] [PubMed]

45. Schlapbach, L.J.; Aebischer, M.; Adams, M.; Natalucci, G.; Bonhoeffer, J.; Latzin, P.; Nelle, M.; Bucher, H.U.; Latal, B.; Swiss Neonatal, N.; et al. Impact of sepsis on neurodevelopmental outcome in a Swiss National Cohort of extremely premature infants. Pediatrics 2011, 128, e348-e357. [CrossRef] [PubMed] 
46. Adams-Chapman, I.; Bann, C.M.; Das, A.; Goldberg, R.N.; Stoll, B.J.; Walsh, M.C.; Sanchez, P.J.; Higgins, R.D.; Shankaran, S.; Watterberg, K.L.; et al. Neurodevelopmental outcome of extremely low birth weight infants with Candida infection. J. Pediatr. 2013, 163, 961-967. [CrossRef]

47. Mitha, A.; Foix-L'Helias, L.; Arnaud, C.; Marret, S.; Vieux, R.; Aujard, Y.; Thiriez, G.; Larroque, B.; Cambonie, G.; Burguet, A.; et al. Neonatal infection and 5-year neurodevelopmental outcome of very preterm infants. Pediatrics 2013, 132, e372-e380. [CrossRef]

48. Synnes, A.; Luu, T.M.; Moddemann, D.; Church, P.; Lee, D.; Vincer, M.; Ballantyne, M.; Majnemer, A.; Creighton, D.; Yang, J.; et al. Determinants of developmental outcomes in a very preterm Canadian cohort. Arch. Dis. Child. 2016, 6. [CrossRef]

49. Bright, H.R.; Babata, K.; Allred, E.N.; Erdei, C.; Kuban, K.C.K.; Joseph, R.M.; O'Shea, T.M.; Leviton, A.; Dammann, O.; Investigators, E.S. Neurocognitive Outcomes at 10 Years of Age in Extremely Preterm Newborns with Late-Onset Bacteremia. J. Pediatr. 2017, 187, 43-49. [CrossRef]

50. Bolisetty, S.; Tiwari, M.; Schindler, T.; Bowen, J.; Bajuk, B.; Carlisle, H.; Kent, A.; Smith, J.; Craven, P.; Glover, R.; et al. Neurodevelopmental outcomes of extremely preterm infants in New South Wales and the Australian Capital Territory. J. Paediatr. Child Health 2018. [CrossRef]

51. Msall, M.E.; Buck, G.M.; Rogers, B.T.; Merke, D.P.; Wan, C.C.; Catanzaro, N.L.; Zorn, W.A. Multivariate risks among extremely premature infants. J. Perinatol. 1994, 14, 41-47.

52. Friedman, S.; Richardson, S.E.; Jacobs, S.E.; O'Brien, K. Systemic Candida infection in extremely low birth weight infants: Short term morbidity and long term neurodevelopmental outcome. Pediatr. Infect. Dis. J. 2000, 19, 499-504. [CrossRef] [PubMed]

53. Hack, M.; Wilson-Costello, D.; Friedman, H.; Taylor, G.H.; Schluchter, M.; Fanaroff, A.A. Neurodevelopment and predictors of outcomes of children with birth weights of less than $1000 \mathrm{~g}$ : 1992-1995. Arch. Pediatr. Adolesc. Med. 2000, 154, 725-731. [CrossRef] [PubMed]

54. Hoekstra, R.E.; Ferrara, T.B.; Couser, R.J.; Payne, N.R.; Connett, J.E. Survival and long-term neurodevelopmental outcome of extremely premature infants born at 23-26 weeks' gestational age at a tertiary center. Pediatrics 2004, 113, e1-e6. [CrossRef]

55. Shah, D.K.; Doyle, L.W.; Anderson, P.J.; Bear, M.; Daley, A.J.; Hunt, R.W.; Inder, T.E. Adverse neurodevelopment in preterm infants with postnatal sepsis or necrotizing enterocolitis is mediated by white matter abnormalities on magnetic resonance imaging at term. J. Pediatr. 2008, 153, 170-175. [CrossRef] [PubMed]

56. Jang, D.H.; Sung, I.Y.; Jeon, J.Y.; Moon, H.J.; Kim, K.S.; Kim, E.A.R.; Lee, B.S. Neurodevelopmental outcomes in very low-birth-weight infants in Korea: 1998-2007 vs. 1989-1997. J. Child Neurol. 2011, 26, 1405-1410. [CrossRef] [PubMed]

57. Alshaikh, B.; Yee, W.; Lodha, A.; Henderson, E.; Yusuf, K.; Sauve, R. Coagulase-negative staphylococcus sepsis in preterm infants and long-term neurodevelopmental outcome. J. Perinatol. 2014, 34, 125-129. [CrossRef]

58. Hentges, C.R.; Silveira, R.C.; Procianoy, R.S.; Carvalho, C.G.; Filipouski, G.R.; Fuentefria, R.N.; Marquezotti, F.; Terrazan, A.C. Association of late-onset neonatal sepsis with late neurodevelopment in the first two years of life of preterm infants with very low birth weight. J. Pediatr. (Rio J.) 2014, 90, 50-57. [CrossRef]

59. Yang, P.; Chen, Y.H.; Yen, C.F.; Chen, H.L. Psychiatric diagnoses, emotional-behavioral symptoms and functional outcomes in adolescents born preterm with very low birth weights. Child Psychiatry Hum. Dev. 2015, 46, 358-366. [CrossRef]

60. Maruyama, H.; Nakata, Y.; Kanazawa, A.; Kikkawa, K. Survival and Neurodevelopmental Outcomes of Very Low Birth Weight Infants in a Regional Core Hospital in Kochi, Japan. Acta Med. Okayama 2016, 70, 345-352.

61. Young, J.M.; Morgan, B.R.; Powell, T.L.; Moore, A.M.; Whyte, H.E.A.; Smith, M.L.; Taylor, M.J. Associations of Perinatal Clinical and Magnetic Resonance Imaging Measures with Developmental Outcomes in Children Born Very Preterm. J. Pediatr. 2016, 170, 90-96. [CrossRef]

62. Zonnenberg, I.A.; van Dijk-Lokkart, E.M.; van den Dungen, F.A.M.; Vermeulen, R.J.; van Weissenbruch, M.M. Neurodevelopmental outcome at 2 years of age in preterm infants with late-onset sepsis. Eur. J. Pediatr. 2019. [CrossRef] [PubMed]

63. Lee, B.E.; Cheung, P.Y.; Robinson, J.L.; Evanochko, C.; Robertson, C.M. Comparative study of mortality and morbidity in premature infants (birth weight, $<1250 \mathrm{~g}$ ) with candidemia or candidal meningitis. Clin. Infect. Dis. 1998, 27, 559-565. [CrossRef] [PubMed] 
64. Van der Ree, M.; Tanis, J.C.; Van Braeckel, K.N.; Bos, A.F.; Roze, E. Functional impairments at school age of preterm born children with late-onset sepsis. Early Hum. Dev. 2011, 87, 821-826. [CrossRef] [PubMed]

65. de Haan, T.R.; Beckers, L.; de Jonge, R.C.; Spanjaard, L.; van Toledo, L.; Pajkrt, D.; van Wassenaer-Leemhuis, A.G.; van der Lee, J.H. Neonatal gram negative and Candida sepsis survival and neurodevelopmental outcome at the corrected age of 24 months. PLoS ONE 2013, 8, e59214. [CrossRef]

66. Dilli, D.; Eras, Z.; Dilmen, U.; Sakrucu, E.D. Neurodevelopmental evaluation of very low birth weight infants with sepsis at 18 to 24 months corrected age. Indian Pediatr. 2013, 50, 327-330. [CrossRef]

67. Tin, W.; Fritz, S.; Wariyar, U.; Hey, E. Outcome of very preterm birth: Children reviewed with ease at 2 years differ from those followed up with difficulty. Arch. Dis. Child. Fetal Neonatal Ed. 1998, 79, F83-F87. [CrossRef]

68. Callanan, C.; Doyle, L.; Rickards, A.; Kelly, E.; Ford, G.; Davis, N. Children followed with difficulty: How do they differ? J. Paediatr. Child Health 2001, 37, 152-156. [CrossRef]

69. Wynn, J.L.; Wong, H.R.; Shanley, T.P.; Bizzarro, M.J.; Saiman, L.; Polin, R.A. Time for a neonatal-specific consensus definition for sepsis. Pediatr. Crit. Care Med. 2014, 15, 523-528. [CrossRef]

70. Anderson, P.J.; De Luca, C.R.; Hutchinson, E.; Roberts, G.; Doyle, L.W.; Victorian Infant Collaborative, G. Underestimation of developmental delay by the new Bayley-III Scale. Arch. Pediatr. Adolesc. Med. 2010, 164, 352-356. [CrossRef]

71. Moore, T.; Johnson, S.; Haider, S.; Hennessy, E.; Marlow, N. Relationship between test scores using the second and third editions of the Bayley Scales in extremely preterm children. J. Pediatr. 2012, 160, 553-558. [CrossRef]

72. Luttikhuizen dos Santos, E.S.; de Kieviet, J.F.; Konigs, M.; van Elburg, R.M.; Oosterlaan, J. Predictive value of the Bayley scales of infant development on development of very preterm/very low birth weight children: A meta-analysis. Early Hum. Dev. 2013, 89, 487-496. [CrossRef] [PubMed]

73. Liberati, A.; Altman, D.G.; Tetzlaff, J.; Mulrow, C.; Gotzsche, P.C.; Ioannidis, J.P.; Clarke, M.; Devereaux, P.J.; Kleijnen, J.; Moher, D. The PRISMA statement for reporting systematic reviews and meta-analyses of studies that evaluate health care interventions: Explanation and elaboration. PLoS Med. 2009, 6, e1000100. [CrossRef] [PubMed] 